\title{
Simplest description of charge propagation in a strong background
}

\author{
Martin Lavelle® and David McMullan๑ \\ Centre for Mathematical Sciences, University of Plymouth, Plymouth PL4 8AA, United Kingdom
}

(Received 17 November 2020; accepted 27 January 2021; published 19 February 2021)

\begin{abstract}
Exploiting the gauge freedom associated with the Volkov description of a charge propagating in a plane wave background, we identify a new type of gauge choice which significantly simplifies the theory. This allows us to develop a compact description of the propagator for both scalar and fermionic matter, in a circularly polarized background. It is shown that many of the usually observed structures are gauge artifacts. We then analyze the full ultraviolet behavior of the one-loop corrections for such charges. This enables us to identify and contrast the different renormalization prescriptions needed for both types of matter.
\end{abstract}

DOI: 10.1103/PhysRevD.103.036015

\section{INTRODUCTION}

Very early in the development of quantum electrodynamics, QED, it was understood that the interaction of light with matter was best described in a way that introduced extra, unphysical, degrees of freedom $[1,2]$. The expected two components of the photon, at each spacetime point, were embedded in the four components of the vector potential, as these were needed to formulate the interaction with matter. The recovery of physical results then followed from the gauge invariance of QED. Gauge fixing allows for a direct recovery of the physical dynamics of the theory. This is the case both for interactions in the vacuum and in a background. In the Volkov description of the propagation of matter through a plane wave background [3], there is also an implicit gauge fixing for the background field. Such propagation has been studied at both tree-level and with loop corrections, see for example [4,5].

Counting degrees of freedom in such gauge theories is complicated by the Lorentzian signature of spacetime. The naive expectation would be that two gauge fixing conditions are needed to remove the two extra degrees of freedom, but in practice a single covariant gauge suffices to define photon propagators and hence S-matrix elements. Unitarity arguments can then show that suitably defined cross sections between appropriate states correspond to physical results with the correct degrees of freedom.

For propagation in a background, the gauge freedom in describing the background potential is, as noted above, often implicit in the formalism. That is, the explicit form of

Published by the American Physical Society under the terms of the Creative Commons Attribution 4.0 International license. Further distribution of this work must maintain attribution to the author(s) and the published article's title, journal citation, and DOI. Funded by SCOAP . the potential implies that a gauge fixing condition has been used. So for the plane wave situation described by the Volkov solution, the scalar product of the null momentum, pointing along the beam, with the background potential vanishes. This is essentially a light cone gauge choice for the potential. There is still some residual gauge freedom in the choice of the background potential, but there is no fundamental requirement for adding an additional gauge fixing condition on the background.

However, the Volkov solution is very complicated and disentangling physics from flotsam is a challenge. In this paper we will argue that a specific choice of additional gauge fixing on the background can significantly simplify the description of both the classical and quantum propagation of a charge through the background. We shall see that this holds for both weak and strong backgrounds, and leads to clear renormalization conditions on the fields and physical parameters. This will be shown for both scalar and fermionic matter, and in this way we will be able to highlight and contrast some of the simple results found here for the renormalization of both theories through the use of our additional gauge fixing condition on the background.

The approach taken here is perturbative in the strength of the background, and in that way we can build upon the familiar and precise language of perturbative quantum field theory. We will thus be able to explicitly introduce counterterms and renormalize using standard field theory constructions. That this can be done for both types of matter and for both weak and strong backgrounds, points to the great utility of imposing our additional gauge fixing condition on the background. This paper will take the background to be circularly polarized, as that choice will lead to the simplest possible expressions for the propagator, especially in the context of scalar matter.

The plan of this paper is to first discuss, in Sec. II, the background gauge freedom and introduce the additional, 
momentum gauge choice on it. Then, in Sec. III, we couple the background to scalar matter. The great utility of the momentum gauge is demonstrated here as we will be able to present a full and self-contained account of the propagation, and its one-loop ultraviolet corrections, of such matter in both a weak and strong background. As well as being of great interest in its own right, this will allow us to introduce some of the key arguments and notation that will then be refined when we treat the case of fermionic matter in the rest of the paper. In this section, and throughout the paper, we regularize ultraviolet divergences by using dimensional regularization with $D=4-2 \varepsilon$.

In Sec. IV we consider fermionic matter and introduce the key ingredients needed to describe its interaction with the background. Then, in Sec. V, we derive the full treelevel fermionic propagator in the background, and see again how the additional momentum gauge choice greatly simplifies this derivation. The one-loop corrections to the propagator in a weak background are presented in Sec. VI, and these are extended to the full, strong background, oneloop calculations in Sec. VII. Armed with these results, we then go on to discuss the renormalization of both the scalar and fermionic theories in Sec. VIII. We then conclude this paper in Sec. IX, where we also discuss how the approach taken here can be extended to other polarization choices for the background. Some key technical results are given in the appendixes.

\section{BACKGROUND FIELD GAUGE FREEDOM}

The real classical potential, $A_{\mathrm{c}}^{\mu}$, describing a circularly polarized background is most conveniently written as the sum of two conjugate fields:

$$
A_{\mathrm{c}}^{\mu}=\mathcal{A}^{\mu}+\mathcal{A}^{* \mu}
$$

where the complex potential is given by

$$
\mathcal{A}^{\mu}=\frac{1}{2} e\left(a_{1}^{\mu}+i a_{2}^{\mu}\right) \mathrm{e}^{-i x \cdot k} .
$$

Here $k^{\mu}$ is the null momentum characterizing the plane wave background, $a_{1}^{\mu}$ and $a_{2}^{\mu}$ are orthogonal, real, spacelike vectors which satisfy the common normalization condition that $a^{2}:=a_{1} \cdot a_{1}=a_{2} \cdot a_{2}<0$. This complex potential also satisfies the null gauge condition

$$
k \cdot \mathcal{A}=0,
$$

which is equivalent to the two real conditions that $k \cdot a_{1}=k \cdot a_{2}=0$.

It should be noted that choosing a particular direction along which the background points introduces both a directional, $x$, and momentum, $k$, dependence to the potential $\mathcal{A}^{\mu}$, as is clear from the final term in (2). We shall soon see, though, that this potential is essentially the background interaction term in a perturbative approach to the system, and it is for that reason that we suppress its explicit dependence on these variables. However, as discussed in [6], and shown later here at all orders in perturbation theory, this will still lead to a multiplicative, momentum space renormalization procedure.

It is important to also note that there is still a residual gauge freedom in the potential, since $\mathcal{A}^{\mu}+\Lambda k^{\mu}$ also satisfies the null gauge condition (3) for arbitrary $\Lambda$ with the same spatial dependence as $\mathcal{A}^{\mu}$. In terms of the real potentials, this gauge freedom is $a_{1}^{\mu} \rightarrow a_{1}^{\mu}+\Lambda_{1} k^{\mu}$ and $a_{2}^{\mu} \rightarrow a_{2}^{\mu}+\Lambda_{2} k^{\mu}$, where then $\Lambda=\frac{1}{2} e\left(\Lambda_{1}+i \Lambda_{2}\right) \mathrm{e}^{-i x \cdot k}$.

It is helpful to be a bit more explicit about this residual gauge freedom. If we write $k^{\mu}=\left(k^{0}, 0,0, k^{0}\right)$, then all the conditions on the real potentials are satisfied by writing

$a_{1}=\left(\begin{array}{l}0 \\ \alpha \\ \beta \\ 0\end{array}\right)+\Lambda_{1}\left(\begin{array}{c}k^{0} \\ 0 \\ 0 \\ k^{0}\end{array}\right)$ and $a_{2}= \pm\left(\begin{array}{c}0 \\ \beta \\ -\alpha \\ 0\end{array}\right)+\Lambda_{2}\left(\begin{array}{c}k^{0} \\ 0 \\ 0 \\ k^{0}\end{array}\right)$,

where the common amplitude normalization is $a^{2}=$ $-\alpha^{2}-\beta^{2}$, and the sign ambiguity reflects left or right polarization choices, as discussed in [7].

It is tempting to think of the two parameters $\alpha$ and $\beta$ in (4) as the natural representation of the true degrees of freedom for the background. Indeed, for light by light scattering, such an identification is sensible. But it is not necessarily the best representation of the background when matter is present. We now introduce a new characterization of the true degrees of freedom that is much better suited to calculations involving a charge propagating through the background.

Consider a charge of mass $m$ that has associated with it a timelike momentum $p^{\mu}$ describing its propagation through the background. This momentum may, or may not, be taken to be on-shell. But, given that $k^{\mu}$ is the fixed null momentum associated with the background, we can ensure that $p \cdot k \neq 0$. What is more, in this plane wave description, the momentum $p$ is interpreted as an external momentum and thus is not integrated over in any loop calculation associated with the propagation of the charge. So we are able to ensure that $p \cdot k$ will never vanish in both the treelevel propagator and its loop corrections.

We now impose an additional gauge condition on the background potential by requiring that, as well as (3), we have

$$
p \cdot \mathcal{A}=0
$$

In terms of the explicit representation (4), this momentum gauge condition fixes the residual gauge freedom so that 


$$
\Lambda_{1}=\frac{p_{1} \alpha+p_{2} \beta}{p \cdot k} \quad \text { and } \quad \Lambda_{2}= \pm \frac{p_{1} \beta-p_{2} \alpha}{p \cdot k}
$$

For example, if the charge was static, or moving solely along the $z$ axis, so that $p=\left(p_{0}, 0,0, \lambda p_{0}\right)$ with $|\lambda|<1$, then $\Lambda_{1}=\Lambda_{2}=0$, and we have the very natural representation mentioned earlier with $a_{1}^{\mu}$ and $a_{2}^{\mu}$ only having components in the transverse directions to the background.

But now, suppose the particle was moving along the $x$ axis. The momentum can then be written as $p=$ $\left(p_{0}, p_{1}, 0,0\right)$, and we impose the timelike requirement that $p^{2}=p_{0}^{2}-p_{1}^{2}>0$. Then we find, from (4), that

$$
a_{1}=\left(\begin{array}{c}
\frac{p_{1} \alpha}{p_{0}} \\
\alpha \\
\beta \\
\frac{p_{1} \alpha}{p_{0}}
\end{array}\right) \quad \text { and } \quad a_{2}= \pm\left(\begin{array}{c}
\frac{p_{1} \beta}{p_{0}} \\
\beta \\
-\alpha \\
\frac{p_{1} \beta}{p_{0}}
\end{array}\right)
$$

We shall see that these simple examples, and their full timelike extensions, give a computationally efficient way to characterize the background field for such a propagating charge.

Note that the above mentioned static class of representations of the background potential could also be characterized by the additional light cone condition that $\bar{k} \cdot \mathcal{A}=0$, where $\bar{k}$ points along the dual light cone direction: $\bar{k}^{\mu}=\left(k^{0}, 0,0,-k^{0}\right)$. This choice also ensures that $k \cdot \bar{k} \neq 0$ as it is just the $\lambda \rightarrow-1$ limit of our earlier static class. In applications to light by light scattering, there is also great utility in this additional gauge choice, see for example [8-10]. However, in the context of particle propagation, we shall see that focusing on the light cone structure obstructs the rich interplay between the lightlike background and the timelike particle dynamics inherent in this system. Exploiting this will lead to significant computational advantages and clearer physical insight in to this complex but important system.

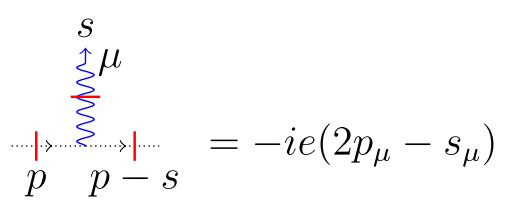

Dotted lines are used here to represent the scalar propagators while wavy lines correspond to the photons.

\footnotetext{
${ }^{1}$ See the discussion on page 90 of [11] concerning such truncated lines and possible representations.
}

Before concluding this general introduction to the kinematics of our system, it is worth noting that the above discussion of the momentum gauge choice assumes that it is sensible to talk of the charge as having a given momentum, $p$. Obviously, in the context of scattering, the momentum will change. Any such measurable scattering is not an ambiguity in the formalism, and the momentum gauge can still be used for at least, say, the incoming particle. However, even in the context of simple charge propagation, with no additional external interactions, the background itself obscures any idea of an unambiguous particle momentum.

So, although we have characterized the charge as having momentum $p$, the fact that it is propagating in a background means that the actual momentum is ambiguous. More precisely, we should allow for its momentum to be of the form $p+n k$, where the integer $n$ counts the number of absorptions from the background minus the number of emissions degenerate to the background.

It is important, though, to note that this change in the momentum will not affect the overall gauge fixing conditions being proposed here since, from (3) and (5), we also have $(p+n k) \cdot \mathcal{A}=0$, for all possible values of $n$.

We now begin our analysis of the propagation of matter through this background. Although our primary interest is in fermionic matter, we shall start with the much simpler case of scalar matter. Our choice of polarization and momentum gauge now becomes particularly effective, and the transition to intense backgrounds will be almost immediate. This will be a good test case and help motivate the key definitions needed for the more complex fermionic structures that will be the main focus of this paper.

\section{SCALAR MATTER}

The quadratic nature of the Lagrangian for scalar QED means that the matter interacts with photons via either a three or four point vertex. The Feynman rules for these vertex contributions are given by the truncated ${ }^{1}$ diagrams, i.e., Green's functions with external lines removed as signified by the small bars on them:

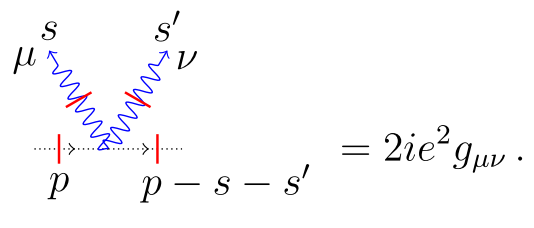

These rules are equivalent to those derived in, for example, Sec. 6-1-4 of [12].

When the photon is taken to be degenerate with the plane wave background, these vertex terms can be contracted with suitably normalized products of the background potential, (1), to give the background 
interactions with the scalar matter in terms of either absorptions

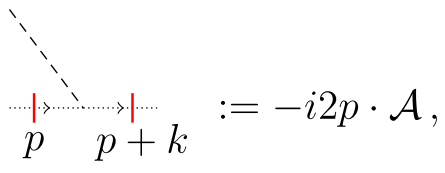

or emissions

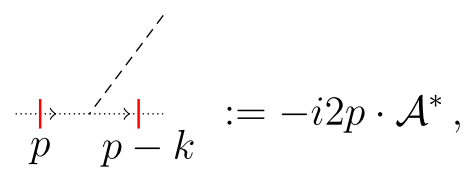

along with the various mixed absorption or emission, seagull interactions

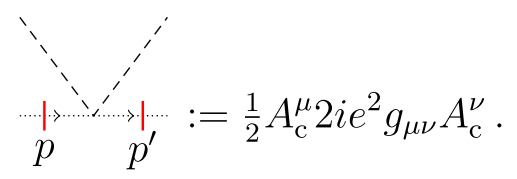

Our choice of momentum gauge, (5), then greatly simplifies these interactions as both the absorption, (9), and emission, (10), interactions vanish. In addition, our choice of circular polarization, (2), means that both $\mathcal{A}$. $\mathcal{A}=0$ and $\mathcal{A}^{*} \cdot \mathcal{A}^{*}=0$, and we quickly see that the only surviving seagull interaction is the momentum conserving, $p^{\prime}=p$, one with Feynman rule

$$
\frac{1}{2} A_{\mathrm{c}}^{\mu} 2 i e^{2} g_{\mu \nu} A_{\mathrm{c}}^{\nu}=2 i \mathcal{A}^{*} \cdot \mathcal{A}=i e^{2} a^{2} .
$$

From the discussion preceding (3), we see that the background amplitude satisfies a spacelike normalization condition, $a^{2}<0$. It is thus useful to introduce the positive scalar quantity, $\mathfrak{m}>0$, defined by

$$
\mathfrak{m}^{2}=-e^{2} a^{2} .
$$

Hence we see that, by using the momentum gauge (5), the sole surviving interaction of the scalar matter with the circularly polarized background is given by the simple seagull term:

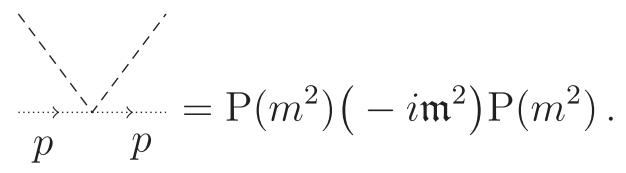

Note that we have introduced in this last expression a useful, compact notation for the scalar propagator that emphasizes its mass dependence, so that

$$
\mathrm{P}\left(m^{2}\right):=\frac{i}{p^{2}-m^{2}+i \epsilon} .
$$

Two such seagull interactions are then given by

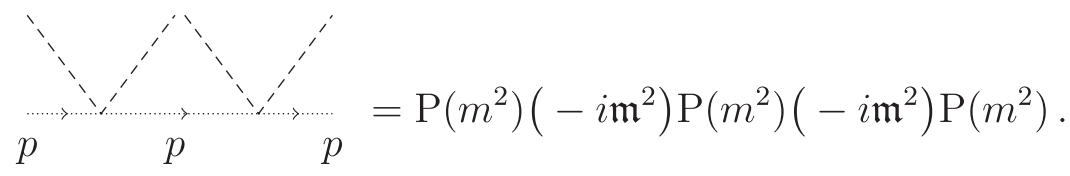

From this we see that multiple interactions with the background are now simple products of these seagulls. So $r \geq 0$ such interactions can be represented as

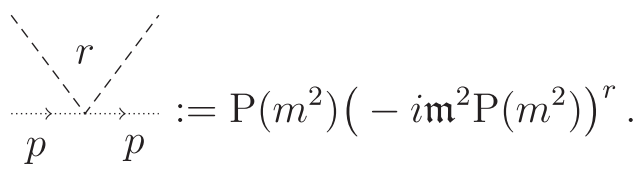

Note that $r=0$ here corresponds to the scalar propagator $\mathrm{P}\left(m^{2}\right)$. When $r=1$ we will often omit the label, as in (14).

Summing this last result over all possible values for $r$ will then describe the physical propagation of the scalar charge through the background. It is important to be able to distinguish the resulting all orders propagation from the usual, perturbative, vacuum propagation of the charge. The convention adopted for a long time, as can be seen in
Sec. 105 of [13], was to use a thicker line to represent the propagator in the background. However, there has been a trend in recent years to make visually clearer the distinct types of propagation being considered in these complex systems. This can be seen in, for example, Fig. 6 in [14] and Fig. 1 in [15], where a double line was used to distinguish propagation in the background.

We hence define the scalar double line propagator of momentum $p$, in the momentum gauge (5), by

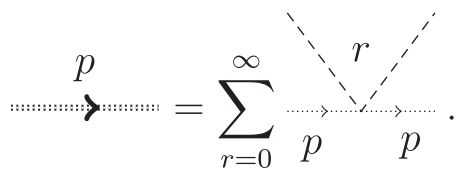

Thus we have the simple result, which follows immediately from (17), that 


$$
\stackrel{p}{>\cdots \cdots \cdots}=\mathrm{P}\left(m^{2}+\mathfrak{m}^{2}\right) .
$$

So, from this strong field summation of the interactions with the background, it is clear that the only impact of the background on the scalar particle is that its mass has increased to $m_{*}^{2}:=m^{2}+\mathfrak{m}^{2}$. This is surprisingly simple. Normally this propagator would involve an infinite sum over different poles (sidebands) and, most strikingly, break translational invariance. None of these complications are present due to our momentum gauge choice.

Having constructed the tree-level propagator for the scalar particle in our momentum gauge fixed background, we now consider its one-loop corrections and the associated ultraviolet structures. This will require an additional gauge fixing choice to be made, but now its role is to allow for the construction of the photon's propagator within the loop, rather than the description of the charge's propagation through the background.

In order to understand the impact of this gauge choice on the one-loop structure, we will take the photon's propagator, $D_{\mu \nu}(s)$, to be in the full Lorentz class of gauges, as given by

$$
D_{\mu \nu}(s)=\frac{-i}{s^{2}+i \epsilon}\left(g_{\mu \nu}+(\xi-1) \frac{s_{\mu} s_{\nu}}{s^{2}}\right) .
$$

We recall that this class includes the Feynman gauge, where $\xi=1$, and Landau (or Lorenz) gauge when $\xi=0$.

In addition to gauge fixing, loops require a method to regularize the ultraviolet sector, and for that we adopt dimensional regularization. So we take the spacetime dimension to be $D=4-2 \varepsilon$, with $\varepsilon>0$, and introduce a mass scale, $\mu$, to maintain the canonical dimensions for the loop integral and renormalized fields.

As expected, loop corrections to the scalar propagator in the background will contain ultraviolet divergences, but these are now worse than those encountered in the fermionic theory. In addition to the logarithmic divergences, we now also get quadratic ones. The great attraction to using dimensional regularization is that it deals with these polynomial types of ultraviolet structures in a very efficient way by putting them equal to zero. But some care is needed in doing that as we can also encounter other classes of divergences that can interfere with this prescription.

The simplicity of dimensional regularization can be seen most dramatically in the seagull loop diagram given by

$$
\underset{p \text { s. }}{p}=\mathrm{P}\left(m^{2}\right) 2(D+\xi-1) e^{2} \mu^{2 \varepsilon} \int d^{D} s \frac{1}{s^{2}} \mathrm{P}\left(m^{2}\right) \text {. }
$$

By simple power counting, we see that the loop integral in (21) diverges quadratic in the large $s$, ultraviolet sector. But, within dimensional regularization, this integral can be evaluated for some $D<4$ and then analytically continued back to four dimensions. The end result is that the integral vanishes. Thus there is no contribution to the propagator from the one-loop term (21).

The nonvanishing one-loop correction to the scalar propagator is thus given by the self-energy term

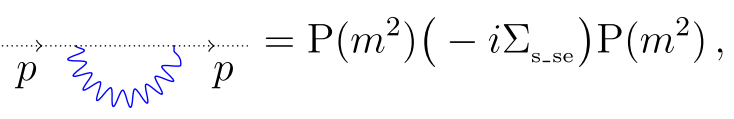

where the scalar's self-energy is given at one-loop by

$$
\begin{aligned}
-i \Sigma_{\text {S_se }}= & -e^{2} \mu^{2 \varepsilon} \int \mathrm{d}^{D} s\left(\frac{(p+s)^{2}}{\left(s^{2}-m^{2}\right)(s-p)^{2}}\right. \\
& \left.+(\xi-1) \frac{\left(p^{2}-s^{2}\right)\left(p^{2}-s^{2}\right)}{\left(s^{2}-m^{2}\right)(s-p)^{2}(s-p)^{2}}\right)
\end{aligned}
$$

and we have suppressed for brevity the $i \epsilon$ prescription for the poles of the various propagators within the loop.

Again we see quadratic divergences here coming from the $s^{2}$ term in the first numerator and the $s^{2} s^{2}$ factor in the second. But in addition, the second term also produces a subleading, logarithmic divergence. Factorizing the numerators so as to cancel terms in the denominators leads to finite parts, which we are not considering in this paper, plus the ultraviolet divergent terms:

$$
\begin{aligned}
-i \Sigma_{\mathrm{S} \_\mathrm{se}}= & -e^{2} \mu^{2 \varepsilon} \int \mathrm{d}^{D} s\left(\frac{p^{2}+m^{2}+2 p \cdot s}{\left(s^{2}-m^{2}\right)(s-p)^{2}}\right. \\
& \left.+\xi \frac{1}{s^{2}}-(\xi-1) \frac{p^{2}-m^{2}}{s^{2} s^{2}}\right) .
\end{aligned}
$$

The important point to note here is that, as with the seagull, the quadratically divergent term is purely ultraviolet in nature, and can thus be robustly set to zero within dimensional regularization. But the final, logarithmic divergence, tadpole is more subtle as it diverges in both the ultraviolet (regularized by taking $\varepsilon>0$ ) and infrared (regularized by taking $\varepsilon<0$ ) regimes. Extracting its ultraviolet divergence now leads to a nonvanishing, gauge dependent contribution to the self-energy for the scalar particle. The end result is that the self-energy term (22) has a gauge dependent ultraviolet pole contribution, $-i \Sigma_{\mathrm{s}_{\mathrm{Se}} \text {, which can be written }}^{\mathrm{UV}}$ in terms of the mass and inverse propagator as

$$
-i \Sigma_{\mathrm{S}_{-} \mathrm{Se}}^{\mathrm{UV}}=-i \frac{e^{2}}{(4 \pi)^{2}}\left(3 m^{2}-i(\xi-3) \mathrm{P}\left(m^{2}\right)^{-1}\right) \frac{1}{\varepsilon}
$$

The vertex correction to the lowest order scalar interaction with the background, (14), is then given by 


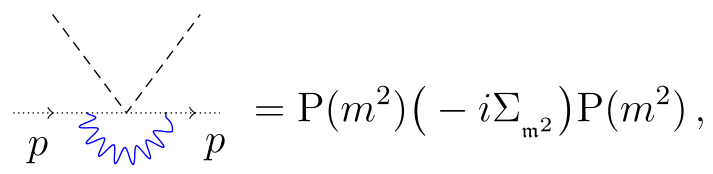

where now the loop correction to the induced mass is

$$
\begin{aligned}
-i \Sigma_{\mathfrak{m}^{2}}= & -e^{2} \mathfrak{m}^{2} \mu^{2 \varepsilon} \int \mathrm{d}^{D} s\left(\frac{(p+s)^{2}}{\left(s^{2}-m^{2}\right)^{2}(s-p)^{2}}\right. \\
& \left.+(\xi-1) \frac{\left(p^{2}-s^{2}\right)\left(p^{2}-s^{2}\right)}{\left(s^{2}-m^{2}\right)^{2}(s-p)^{2}(s-p)^{2}}\right) .
\end{aligned}
$$

The extra scalar propagator in the loop here ameliorates the divergences seen in the related self-energy (23), so that we now only have logarithmic terms to deal with. Thus, by naive power counting, both terms in (27) will contribute the same ultraviolet pole, but the second term will have an additional multiplicative factor of $\xi-1$. When combined we see that the overall ultraviolet pole is proportional to the gauge fixing parameter $\xi$. Thus, writing this ultraviolet, double pole correction as $-i \Sigma_{\mathfrak{m}^{2}}^{\mathrm{UV}}$, we have at one-loop the gauge dependent result that

$$
-i \Sigma_{\mathfrak{m}^{2}}^{\mathrm{UV}}=-i \frac{e^{2}}{(4 \pi)^{2}} \xi \mathfrak{m}^{2} \frac{1}{\varepsilon}
$$

The one-loop ultraviolet results (25) and (28), and the expansion of the double line propagator (18), are the only ingredients needed for building the full, one-loop, ultraviolet corrections to the scalar propagator in the background. This claim might seem surprising as we have only considered a single seagull interaction, but we note that if a loop straddles more than one background interaction, then it is ultraviolet finite by simple power counting. This follows immediately from the extension of (27) to that situation, where the power of $\left(s^{2}-m^{2}\right)$ in the denominator will then be greater than two. This means that we only need to consider loops spanning single background seagulls in order to extract the ultraviolet terms.

An immediate consequence of this, single seagull within a loop, result is a simple inductive characterization of the loop corrections to multiple seagulls. We thus have the ultraviolet, loop factorization identity that, for $r \geq 1$,

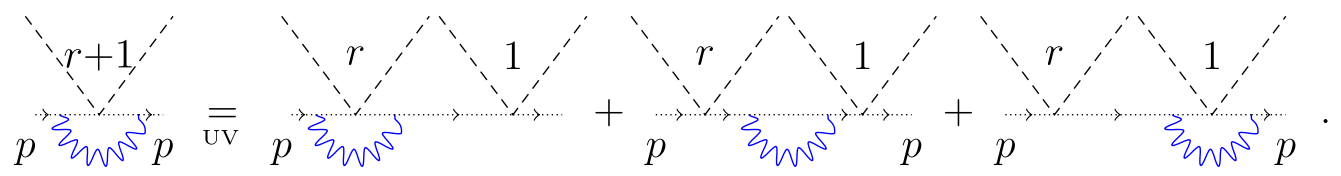

From this it follows by simple induction that if we define $\Delta(r)$, for $r \geq 1$, by

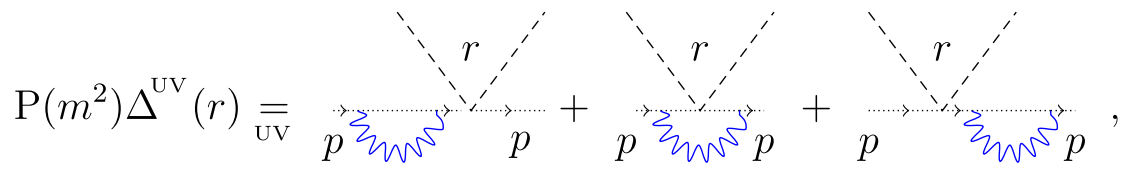

then

$$
\Delta^{\mathrm{UV}}(r)=(r+1)\left(-i \mathfrak{m}^{2} \mathrm{P}\left(m^{2}\right)\right)^{r}\left(-i \Sigma_{\mathrm{S}_{s} \mathrm{e}}^{\mathrm{UV}} \mathrm{P}\left(m^{2}\right)\right)+r\left(-i \mathfrak{m}^{2} \mathrm{P}\left(m^{2}\right)\right)^{r-1}\left(-i \Sigma_{\mathfrak{m}^{2}}^{\mathrm{UV}} \mathrm{P}\left(m^{2}\right)\right) .
$$

Writing $\Delta^{\mathrm{UV}}(0)=-i \sum_{\mathrm{s}_{-} \mathrm{ee}}^{\mathrm{UV}} \mathrm{P}\left(m^{2}\right)$, we then see that summing over all such degenerate processes yields the strong field, oneloop corrections

$$
\begin{aligned}
\mathrm{P}\left(m^{2}\right) \sum_{r=0}^{\infty} \Delta^{\mathrm{UV}}(r) & =\mathrm{P}\left(m^{2}\right) \sum_{r=1}^{\infty} r\left(-i \mathfrak{m}^{2} \mathrm{P}\left(m^{2}\right)\right)^{r-1}\left(-i\left(\Sigma_{\mathrm{S}_{-} \mathrm{se}}^{\mathrm{UV}}+\Sigma_{\mathfrak{m}^{2}}^{\mathrm{UV}}\right)\right) \mathrm{P}\left(m^{2}\right) \\
& =\left(\mathrm{P}\left(m^{2}\right) \sum_{j=0}^{\infty}\left(-i \mathfrak{m}^{2} \mathrm{P}\left(m^{2}\right)\right)^{j}\right)^{2}\left(-i\left(\Sigma_{\mathrm{S}_{-} \mathrm{se}}^{\mathrm{UV}}+\Sigma_{\mathfrak{m}^{2}}^{\mathrm{UV}}\right)\right) .
\end{aligned}
$$

Recognizing in this expression the double line expansions (18) squared allows us to write (32) as the double line self-energy:

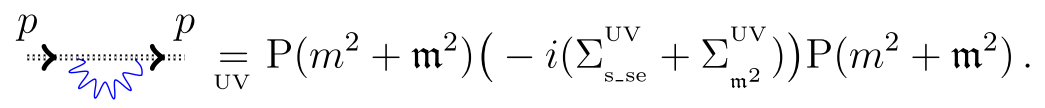


This last result is a very succinct and attractive summary of the ultraviolet, one-loop structure of the scalar QED propagator in our background. Although it was built up perturbatively in the background interactions, it is an all orders result and thus valid for both weak and strong background fields. Through the use of the momentum gauge to remove irrelevant clutter, we have recovered a very simple result that clearly identifies the ultraviolet divergences that need renormalizing in this scalar theory. Indeed, we see here a direct and simple link between the double line representation of a loop contribution and the precise algebraic structure of the corresponding Green's function.

Having obtained this compact result for the scalar theory in the background which is structurally identical to that found in a vacuum, we can now introduce counterterms and renormalize using the familiar techniques of QED. But, before doing so, we shall first analyze what happens with fermionic matter. We will then return to discuss the renormalization of both theories in Sec. VIII.

\section{FERMIONIC MATTER}

The impact of the plane wave background on fermionic matter includes a mass shift to $m_{*}^{2}$, first identified in [3,16], which was later seen to have a more subtle, matrix structure in [17]. The background also generates an infinite class of sidebands that permeate the theory $[6,7,18,19]$, resulting in momentum shifts and additional spacetime phases. We will see, though, that the use of the momentum gauge streamlines the route to the induced mass and also reduces the sidebands to a finite number.

To lay the groundwork for these results, and their oneloop extensions, we will first, in this section, introduce the matrix structures associated with the fermions and identify the key simplifications that follow from the use of the momentum gauge and our choice of polarization. We will then apply these results in the following sections to both the tree and one-loop descriptions of the full propagator in our background.
The absorption by an electron of a photon from the background is now characterized by the absorption matrix, $\mathrm{A}$, which is given in terms of the complex potential, $\mathcal{A}^{\mu}$, by

$$
\mathrm{A}=-i \mathcal{A}
$$

The dual matrix, E, describing the emission of a photon degenerate to the background, is then given by

$$
\mathrm{E}=-i \mathcal{A}^{*}
$$

These are the fermionic counterparts to the scalar terms (9) and (10), but they do not now vanish in the momentum gauge. Indeed, these are now the only interactions with the background for the fermion, as there is no equivalent to the seagull term that was central to the scalar theory.

In terms of these absorption and emission matrices, the gauge conditions (3) and (5) imply the anticommutation results that

$$
\not k \mathrm{~A}=-\mathrm{A} \not k, \quad \not k \mathrm{E}=-\mathrm{E} \not k,
$$

and

$$
\not p \mathrm{~A}=-\mathrm{A} \not p, \quad \not p \mathrm{E}=-\mathrm{E} \not p .
$$

Just as we did for the scalar field, it is useful to introduce a compact notation for the fermionic propagator, but now it needs to incorporate the degeneracy induced by the background that was alluded to earlier. We thus define, for integer $n$, the shifted fermionic propagator, $\mathrm{P}(m)_{n}$, by

$$
\mathrm{P}(m)_{n}=\frac{i}{\not p+n \not k-m+i \epsilon} .
$$

Diagrammatically, these fermionic propagators will be represented by the usual plain line. Thus the fundamental absorption process from the background is now given by

$$
\underset{p+n k p+(n+1) k}{\stackrel{\ddots}{\hookrightarrow}}=\mathrm{P}(m)_{n+1}(-i \mathcal{A}) \mathrm{P}(m)_{n} \equiv \mathrm{P}(m)_{n+1} \mathrm{AP}(m)_{n}
$$

while the emission process to the background is

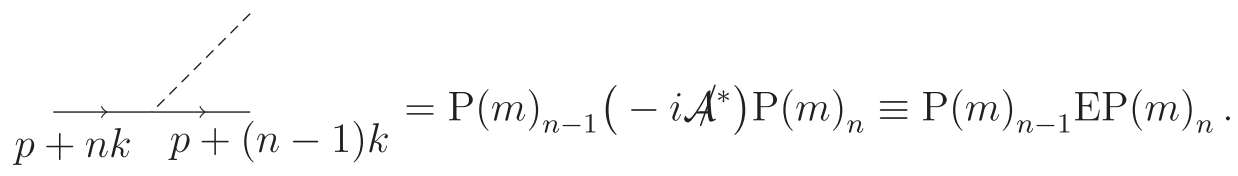


The linear mass dependence and the extra subscript in (38) will help to distinguish this propagator from the scalar one, $\mathrm{P}\left(\mathrm{m}^{2}\right)$, introduced in (15). Obviously, but very importantly, the big technical difference between $\mathrm{P}(m)_{n}$ and $\mathrm{P}\left(\mathrm{m}^{2}\right)$ that must be kept in mind is that the fermionic one is a matrix.

The algebraic complexities of the fermionic theory mean that it will often be useful to abbreviate this fermionic propagator by suppressing the explicit mass term, as in $\mathrm{P}(m)_{n} \rightarrow \mathrm{P}_{n}$. Indeed, we will use this condensed notation for the fermionic propagator in what follows and only reintroduce the more explicit form after Eq. (69).

From our discussion of the scalar theory, we know that the propagator $\mathrm{P}_{0}$ describes both the usual free propagator of momentum $p$ and also the propagator where the number of absorptions equals the number of emissions. Obviously, a similar degeneracy will arise for each propagator, $\mathrm{P}_{n}$. But, in addition, a mismatch between the number of emissions and absorptions will result in a shift in the value of $n$. This process is described using the interactions (34) and (35) in the standard way, by considering the vertex term $\mathrm{P}_{n+1} \mathrm{AP}_{n}$ for an absorption and its dual $\mathrm{P}_{n} \mathrm{EP}_{n+1}$ for an emission. However, what is not standard from a field theory point of view is the fact that these interactions can be rewritten as the difference of two distinct propagators, and that this holds at all orders in the background interactions. This Ward type of identity leads to the sideband description of the charge that was first described in [18] and further refined in [6].

In a perturbative framework, the emergence of sidebands is simply a partial fraction expansion of the absorption, (39), and emission, (40), interactions. This quickly leads to the key absorption and emission identities that

$\mathrm{P}_{n+1} \mathrm{AP}_{n}=\mathrm{IP}_{n}-\mathrm{P}_{n+1} \mathrm{I}$ and $\mathrm{P}_{n-1} \mathrm{EP}_{n}=\mathrm{P}_{n-1} \mathrm{O}-\mathrm{OP}_{n}$.

The existence of this partial fraction decomposition only relies on the light cone property of $k^{\mu}$ and the null gauge condition (3). But the form of the "In" factor, I, and the "Out" factor, $\mathrm{O}$, is sensitive to the momentum gauge choice, (5). We now find that

$$
\mathrm{I}:=\frac{2 p \cdot \mathcal{A}+\not k \mathcal{A}}{2 p \cdot k}=\frac{\not K \mathcal{A}}{2 p \cdot k},
$$

while its dual "Out" matrix is

$$
\mathrm{O}:=\frac{2 p \cdot \mathcal{A}^{*}+\mathcal{A}^{*} \not k}{2 p \cdot k}=\frac{\mathcal{A}^{*} \not k}{2 p \cdot k} .
$$

We have now introduced all the basic variables needed to build up a description of the electron propagating through the background. The way to proceed, that makes the transition to the loop corrections most transparent, is to now shift from the language of absorptions, (34), and emissions, (35), to that of the "In", (42), and "Out", (43), factors. This perturbative refocusing of the formalism for fermions will be the topic of the next section. Prior to embarking on that, though, it is useful to conclude this section with a summary of the key new simplifications that follow from our choice of circular polarization, (2), and the additional momentum gauge condition, (5).

The immediate impact of using a circular polarization is that polynomials in the interactions become trivial. In particular, we have already noted that from (2), $\mathcal{A} \cdot \mathcal{A}=$ $\mathcal{A}^{*} \cdot \mathcal{A}^{*}=0$. This means that the scalar term $v$ and $v^{*}$ that play an important role in the full elliptical class of polarization, see [7], now vanish:

$$
v:=\frac{\mathcal{A} \cdot \mathcal{A}}{2 p \cdot k}=0 \quad \text { and } \quad v^{*}:=\frac{\mathcal{A}^{*} \cdot \mathcal{A}^{*}}{2 p \cdot k}=0 .
$$

In terms of the absorption and emission matrices, these polarization dependent simplifications become

$$
\mathrm{A}^{2}=0 \quad \text { and } \quad \mathrm{E}^{2}=0 .
$$

These last two results are easily extended using the momentum gauge conditions (37), so that

$$
\mathrm{A} \not p \mathrm{~A}=0 \quad \text { and } \quad \mathrm{E} \not p \mathrm{E}=0 .
$$

Written in terms of the propagators (38), these last identities become

$$
\mathrm{AP}_{n} \mathrm{~A}=0 \quad \text { and } \quad \mathrm{EP}_{n} \mathrm{E}=0 .
$$

For the "In" and "Out" factors we have similar algebraic properties to (45), but now these are polarization independent and just follow from the momentum gauge choice, so that

$$
\mathrm{I}^{2}=0 \quad \text { and } \quad \mathrm{O}^{2}=0 .
$$

In addition, the momentum gauge choice also implies the polarization independent, trivial mixed products of these factors:

$$
\mathrm{IO}=0 \quad \text { and } \quad \mathrm{OI}=0 .
$$

The choice of circular polarization combines with the momentum gauge to now give momentum insertion identities similar to (46), namely $\mathrm{I} \not \not \mathrm{I}=0$ and $\mathrm{O} \not p \mathrm{O}=0$. These simple results have two very important refinements that will be repeatedly used and extended in our analysis. If we first view the momentum term here as coming from the propagator (38), then we have

$$
\mathrm{IP}_{n} \mathrm{I}=0 \quad \text { and } \quad \mathrm{OP}_{n} \mathrm{O}=0 .
$$


While if we identify the momentum as part of the inverse propagator $\mathrm{P}_{n}^{-1}=-i(\not \supset+n \not k-m)$, then we have

$$
\mathrm{IP}_{n}^{-1} \mathrm{I}=0 \quad \text { and } \quad \mathrm{OP}_{n}^{-1} \mathrm{O}=0 .
$$

It is important to note that the mixed identities in (49) are not reflected in the product of an absorption and an emission matrix. Indeed, from (2), we have $\mathcal{A}^{*} \cdot \mathcal{A}=$ $\frac{1}{2} e^{2} a^{2}$, which does not vanish. This result is actually polarization independent, see [7]. In terms of the absorption and emission matrices, we write this last key identity as

$$
\mathrm{AE}+\mathrm{EA}=-2 \mathcal{A}^{*} \cdot \mathcal{A}=2 p \cdot \mathscr{M},
$$

where the oxymoronic, "mass null vector" is defined by

$$
\mathscr{M}^{\mu}=-\frac{\mathcal{A}^{*} \cdot \mathcal{A}}{p \cdot k} k^{\mu}
$$

Note that from the scalar mass definition (13), we have the vector mass identity that

$$
\mathfrak{m}^{2}=2 p \cdot \mathscr{M} .
$$

In terms of the "In" and "Out" factors, the null vector mass term arises from the momentum insertions whereby $\mathrm{I} \not \not \mathrm{O}+\mathrm{O} \not \not \mathrm{I}=\mathscr{M}$. Written in terms of the inverse propagator, this becomes

$$
\mathrm{IP}_{n}^{-1} \mathrm{O}+\mathrm{OP}_{n}^{-1} \mathrm{I}=-i \| .
$$

Finally, we note that the propagator identities in (50) have the immediate mass insertion generalizations that, for $r \geq 0$,

$$
\mathrm{IP}_{n}\left(-i M \mathrm{P}_{n}\right)^{r} \mathrm{I}=0 \quad \text { and } \quad \mathrm{OP}_{n}\left(-i M \mathrm{P}_{n}\right)^{r} \mathrm{O}=0 .
$$

\section{TREE-LEVEL PROPAGATION}

Loop corrections to the propagation of the charge are most readily introduced via a perturbative formulation of the treelevel results. We shall now develop such a description, taking full advantage of the simplifications that follow from using the momentum gauge. Armed with these results, we shall then be ready to add one-loop corrections to these all orders interactions with the background.

Building upon our definition of the scalar double line propagator, (18), we define the fermionic double line propagator, of momentum $p$, to be the sum over all possible tree-level, perturbative interactions with the background that starts with momentum $p$,

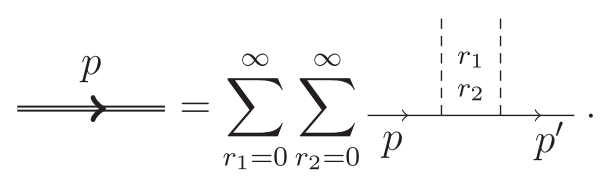

The notation being introduced here is the generalization of the scalar result (18) to the situation where we have both absorptions and emissions from the background. So the sum is now over all processes with $r_{1}$ absorptions and $r_{2}$ emissions, degenerate to the background. In contrast to the scalar theory, each such process will in general correspond to multiple Feynman diagrams. Note that momentum conservation implies that the out going momentum is $p^{\prime}=p+\left(r_{1}-r_{2}\right) k$.

The single absorption process, (39), but in which the charge with initial momentum $p$ absorbs a background photon, is given in terms of the propagator, (38), by the vertex contribution $\mathrm{P}_{1} \mathrm{AP}_{0}$. Thus we have, from (41), the sideband representation of this interaction:

$$
\begin{array}{l:c:c} 
& 1 & \\
& 0 & \\
\hline p & & p+k
\end{array}=\mathrm{IP}_{0}-\mathrm{P}_{1} \mathrm{I} .
$$

In a similar way, the single emission process, (40), becomes

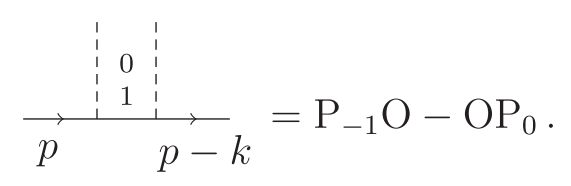

If we now consider two absorptions, then we get from (47) the vanishing result that

$$
\underset{\mathrm{p}}{ } \begin{array}{c:c:c} 
& 2 & \\
& 0 & p+2 k
\end{array}=\mathrm{P}_{2} \mathrm{AP}_{1} \mathrm{AP}_{0}=0 .
$$

This last example can be easily generalized so that if the difference between the number of absorptions and emissions is greater than one, then the contribution to the double line propagator, (57), vanishes:

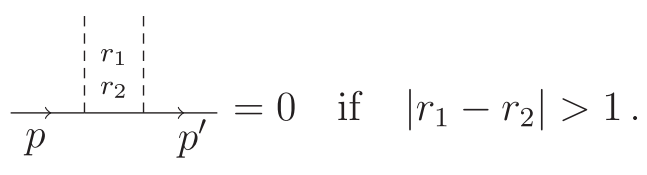

This key vanishing result follows from both our choice of momentum gauge and polarization. The proof is straightforward since all perturbative contributions in (61) must now include parts where we have either two consecutive absorptions or two consecutive emissions, separated by an appropriate propagator $\mathrm{P}_{n}$. These then vanish by the identities (47).

From the vanishing result (61), we see that the only other nonvanishing contributions to the propagator (57) arise when the absorptions alternate with the emissions. The lowest order terms of this form are given by the 
processes whereby the electron first absorbs a photon and then emits back into the background, or first emits then absorbs from the background: $\mathrm{P}_{0}\left(\mathrm{AP}_{-1} \mathrm{E}+\mathrm{EP}_{1} \mathrm{~A}\right) \mathrm{P}_{0}$. The central factor here can be seen as a propagator insertion into the mass term (52). One quickly finds that the momentum gauge implies the polarization independent result that

$$
\mathrm{AP}_{-1} \mathrm{E}+\mathrm{EP}_{1} \mathrm{~A}=-i \dddot{M}+\mathrm{P}_{0}^{-1}\left(\mathrm{IP}_{-1} \mathrm{O}+\mathrm{OP}_{1} \mathrm{I}\right) \mathrm{P}_{0}^{-1}
$$

Hence we see that

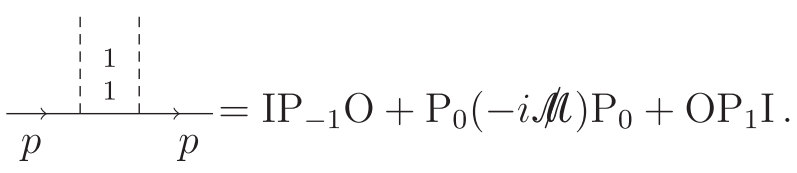

This is the fermionic version of the scalar seagull term (14). Again we note that the fermionic theory has even sidebands associated with this central term.

The lowest order results (58), (59), and (63) can now be extended to all orders in the background interaction. Key to that extension is the following factorization result, that is derived in Appendix A, valid for $r_{1} \geq 0$ and $r_{2} \geq 0$ :

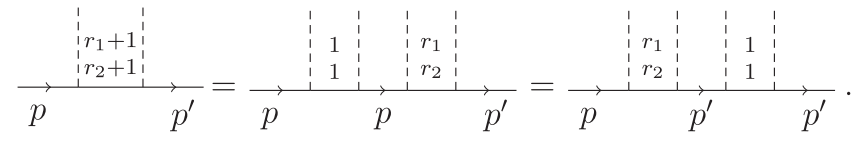

Given the mass generating term in (63), the factorization in (64) will spawn background induced mass terms into all the sidebands seen in (58), (59), and (63). Exploiting this factorization then quickly leads to the results, also discussed in Appendix A, that for all $r \geq 0$ :

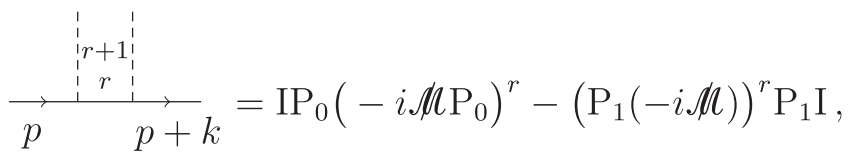

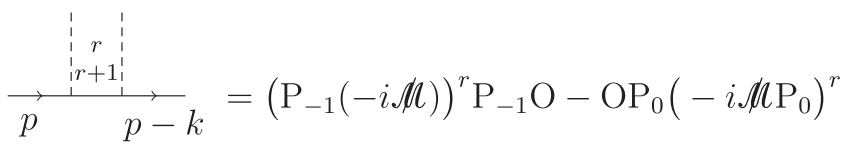

and

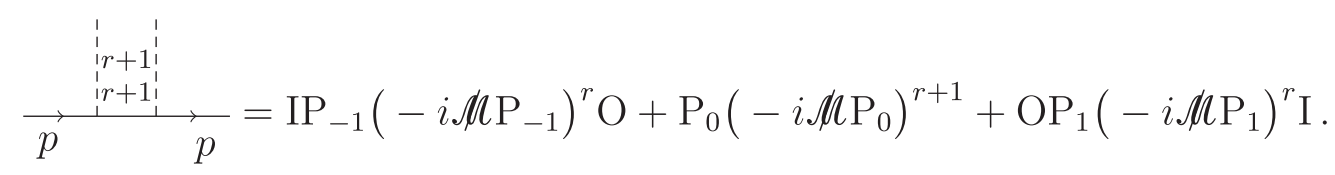

Using the vanishing result (61) in the double line definition (57), we see that the double sum over interactions becomes the single sum:

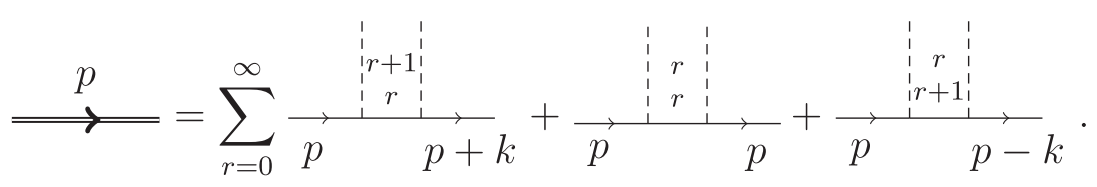

The terms being summed over here are explicitly given by the previous key results (65), (66), and (67).

To interpret this representation of the double line propagator for fermionic matter, it is helpful to reinstate the explicit mass dependence of the propagator, so that $\mathrm{P}_{n} \rightarrow \mathrm{P}(m)_{n}$. Mimicking the scalar argument in (19), if the fermionic mass $m$ is now shifted by the matrix term $\mathscr{M}$, then we get the expansion

$$
\begin{aligned}
\mathrm{P}(m+\mathscr{M})_{n} & =\frac{i}{\not p+n \not k-(m+\mathscr{M})+i \epsilon} \\
& =\sum_{r=0}^{\infty}\left(\mathrm{P}(m)_{n}(-i \mathscr{M})\right)^{r} \mathrm{P}(m)_{n},
\end{aligned}
$$

where in (69) the single propagator term can also be factored out to the left.

Using this mass shift identity allows us to rewrite in a very succinct way the all orders tree-level result (68) as the core sideband expansion: 


$$
\begin{aligned}
\stackrel{p}{\Longrightarrow}= & \mathrm{IP}(m+\boldsymbol{M})_{0}-\mathrm{P}(m+\boldsymbol{M})_{1} \mathrm{I} \\
& +\mathrm{IP}(m+\boldsymbol{M})_{-1} \mathrm{O}+\mathrm{P}(m+\boldsymbol{M})_{0}+\mathrm{OP}(m+\boldsymbol{M})_{1} \mathrm{I} \\
& +\mathrm{P}(m+\boldsymbol{M})_{-1} \mathrm{O}-\mathrm{OP}(m+\boldsymbol{M})_{0} .
\end{aligned}
$$

Note that in this all-orders, tree-level expression for the fermionic double line propagator, the upper terms have spacetime dependence inherited from the "In" factor of $\mathrm{e}^{-i x \cdot k}$, the central terms have no such spacetime factors, while the lower terms inherited from the "Out" factor an $\mathrm{e}^{i x \cdot k}$ dependence.

It is also useful to note that the fermionic propagator $\mathrm{P}(m+\mathscr{M})_{n}$ in these last few expressions can also be partially written in terms of the scalar mass $\mathfrak{m}$, introduced in (13), as

$$
\mathrm{P}(m+\mathscr{M})_{n}=\frac{i(\not p+n \not k+m-\mathscr{M})}{(p+n k)^{2}-\left(m^{2}+\mathfrak{m}^{2}\right)+i \epsilon} .
$$

This representation makes clear the new pole structure in the sidebands and highlights the fundamental difference in this fermionic theory due to the vector nature of the induced mass term in the numerator.

The expression (70) for the all-orders, tree-level, fermionic propagator in the background is surprisingly compact, with a very manageable number of core sidebands. In Appendix B we show how this formulation of the fermionic double line propagator relates to the more standard discussions found in the literature.

\section{ONE-LOOP CORRECTION IN A WEAK BACKGROUND}

Having constructed the tree-level, double line fermionic propagator in (68) and (70), we now want to incorporate into these sideband expressions their one-loop corrections. Just as for the scalar theory, this will be built up perturbatively over the interactions with the background. So we will start our analysis in this section by considering a weak background and hence looking at the loop corrections to the lowest order background terms introduced earlier: the single absorption (58), the single emission (59), and both processes with a single absorption and emission (63). These lowest order one-loop corrections were first discussed in [6], and extended to the full Lorentz class of gauges in [20]. Now we shall exploit the simplifications that arise due to our momentum gauge choice, (5), to give a more direct account of these results for a circularly polarized background.

Using a simple notational extension of the scalar selfenergy term introduced in (22), but now allowing for a sideband momenta of $p+n k$, we take the fermionic selfenergy to be given by the expression

$$
p+\overrightarrow{n k} \xi_{w} \vec{p}+n k=\mathrm{P}_{n}\left(-i \Sigma_{\mathrm{f}-\mathrm{se}}(n)\right) \mathrm{P}_{n} .
$$

Note, though, that by translational invariance, we can focus on the structure of the central sideband here, with $n=0$, and then replace $p$ by $p+n k$ for the more general result in what follows.

We thus have, within our Lorentz class of gauge fixings for the loop,

$$
\begin{aligned}
-i \Sigma_{\mathrm{f}_{-} \mathrm{se}}(0)= & -e^{2} \mu^{2 \varepsilon} \int \mathrm{d}^{D} s\left(\frac{\gamma^{\mu}(\not+m) \gamma_{\mu}}{\left(s^{2}-m^{2}\right)(s-p)^{2}}\right. \\
& \left.+(\xi-1) \frac{(\not-\not \supset)(\not \$+m)(\not \$-\not \supset)}{(s-p)^{2}\left(s^{2}-m^{2}\right)(s-p)^{2}}\right) .
\end{aligned}
$$

In contrast to the equivalent scalar version, (23), there are now no quadratic ultraviolet divergences, but there are still linear and logarithmic ones to be identified. Simple power counting arguments now quickly show that

$$
\begin{aligned}
-i \Sigma_{\mathrm{f}_{-} \mathrm{se}}(0) \underset{\mathrm{UV}}{=} & -e^{2} \int \mathrm{d}^{D} s\left((3 m-(\not p-m)) \frac{1}{s^{2} s^{2}}\right. \\
& \left.-(\xi-1)(\not p-m) \frac{1}{s^{2} s^{2}}\right) .
\end{aligned}
$$

Thus the fermionic version of the ultraviolet scalar result (25), adapted to the $n$th sideband, is the self-energy expression that

$$
-i \Sigma_{\mathrm{f} \_\mathrm{se}}^{\mathrm{UV}}(n)=-i \frac{e^{2}}{(4 \pi)^{2}}\left(3 m-i \xi \mathrm{P}_{n}^{-1}\right) \frac{1}{\varepsilon} .
$$

An analysis of the vertex corrections in the fermionic theory is more involved than in the scalar theory, due to the associated changes in sideband structures related to whether we have an absorption or an emission or both. To unpick this we start with the vertex correction to the fundamental absorption process (58).

The vertex correction to the absorption of an incoming background photon is given by 


$$
\vec{p} \sum_{w p+k}:=\mathrm{P}_{1}\left(-i \Sigma_{\text {in }}\right) \mathrm{P}_{0}
$$

where

$$
\begin{aligned}
- & i \Sigma_{\text {in }} \\
= & -e^{2} \mu^{2 \varepsilon} \int \mathrm{d}^{D} s\left(\frac{\gamma^{\mu}(\not p+\not k+m) \mathcal{A}(\not \supset+m) \gamma_{\mu}}{\left((s+k)^{2}-m^{2}\right)\left(s^{2}-m^{2}\right)(s-p)^{2}}\right. \\
& \left.+(\xi-1) \frac{(\not \$-\not p)(\not \$+\not k+m) \mathcal{A}(\not \not+m)(\not \not-\not h)}{(s-p)^{2}\left((s+k)^{2}-m^{2}\right)\left(s^{2}-m^{2}\right)(s-p)^{2}}\right) .
\end{aligned}
$$

Again, simple power counting arguments quickly show that

$$
-i \Sigma_{\mathrm{in}}=-e^{2} \int \mathrm{d}^{D} s\left(\mathcal{A} \frac{1}{s^{2} s^{2}}+(\xi-1) \mathcal{A} \frac{1}{s^{2} s^{2}}\right) .
$$

Recalling our definition of the absorption matrix, (34), this quickly leads to the ultraviolet, incoming, vertex contribution

$$
-i \Sigma_{\mathrm{in}}^{\mathrm{UV}}:=\frac{e^{2}}{(4 \pi)^{2}} \xi \mathrm{A} \frac{1}{\varepsilon}
$$

Using the sideband representation of the absorption matrix, (41), allows us to rewrite this vertex contribution in terms of consecutive sideband self-energies, (75), so that

$$
-i \Sigma_{\text {in }}^{\mathrm{UV}}=\mathrm{I}\left(-i \Sigma_{\mathrm{f}_{\text {_se }}}^{\mathrm{UV}}(n)\right)-\left(-i \Sigma_{\mathrm{f}_{\text {_se }}}^{\mathrm{UV}}(n+1)\right) \mathrm{I}
$$

To understand the significance of the one-loop results, (75) and (80), we now consider the full, one-loop corrections to the single absorption process as given by the two self-energy and one vertex corrections:

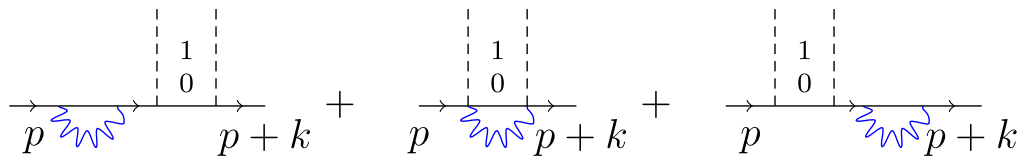

$$
\begin{aligned}
& =\left(\mathrm{IP}_{0}-\mathrm{P}_{1} \mathrm{I}\right)\left(-i \Sigma_{\mathrm{f}_{-} \mathrm{se}}^{\mathrm{UV}}(0)\right) \mathrm{P}_{0}+\mathrm{P}_{1}\left(-i \Sigma_{\text {in }}^{\mathrm{UV}}\right) \mathrm{P}_{0} \\
& +\mathrm{P}_{1}\left(-i \Sigma_{\mathrm{f}_{-} \mathrm{se}}^{\mathrm{UV}}(1)\right)\left(\mathrm{IP}_{0}-\mathrm{P}_{1} \mathrm{I}\right) \\
& =\mathrm{IP}_{0}\left(-i \Sigma_{\mathrm{f}_{-} \mathrm{se}}^{\mathrm{UV}}(0)\right) \mathrm{P}_{0}-\mathrm{P}_{1}\left(-i \Sigma_{\mathrm{f}_{-} \mathrm{se}}^{\mathrm{UV}}(1)\right) \mathrm{P}_{1} \mathrm{I} \text {. }
\end{aligned}
$$

This we recognize as the naively expected, one-loop self-energy corrections to the sidebands in (58).

In a very similar way, the leading one-loop vertex correction to the dual emission process, (59), is given by

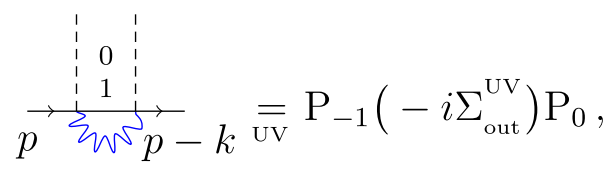

where the outgoing version of the incoming vertex, (79), and its sideband representation (80), are now

$$
-i \Sigma_{\mathrm{out}}^{\mathrm{UV}}=\frac{e^{2}}{(4 \pi)^{2}} \xi \mathrm{E} \frac{1}{\varepsilon}=\left(-i \Sigma_{\mathrm{f}_{-} \mathrm{se}}^{\mathrm{UV}}(n-1)\right) \mathrm{O}-\mathrm{O}\left(-i \Sigma_{\mathrm{f}_{-} \mathrm{se}}^{\mathrm{UV}}(n)\right) .
$$

Hence we quickly see that the one-loop corrections to the single emission process are

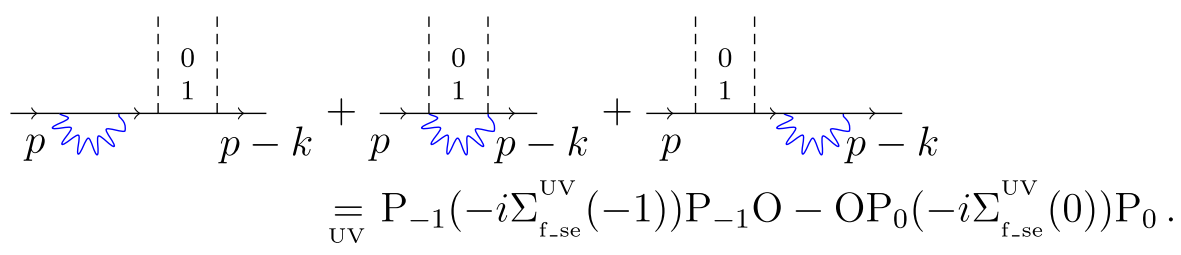

This is also the expected, one-loop self-energy corrections to the absorption sidebands in (59). 
Loop corrections to processes with a mixture of emissions and absorptions introduce an additional, but gauge dependent, ultraviolet divergence associated with the background induced mass term, (53). This was first identified at lowest order in the background, for Feynman gauge, in [6] and then extended to the full Lorentz class in [20]. We have already seen here, in expression (63), that the identification of the background induced mass is simplified by the use of the momentum gauge. Now we will see how that gauge also streamlines the discussion of this new ultraviolet correction.
The one-loop corrections to the lowest order mixed absorption and emission process, (63), can clearly spawn simple self-energy terms. But these corrections can also straddle more complex, interaction structures associated with the background. Indeed, the vertex term here is now a mixture of emissions and absorptions, and the loop corrections are thus more involved. Focusing, though, on the ultraviolet structure leads to a simple factorization of these vertex corrections, the details of which are discussed in Appendix C. One finds

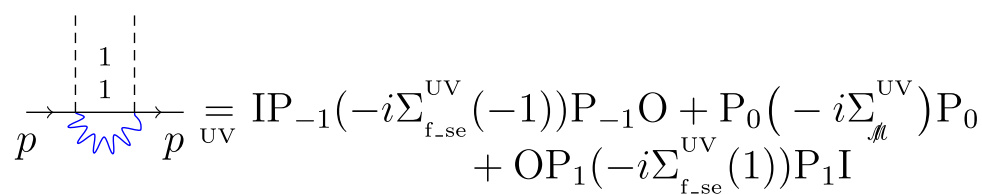

$$
\begin{aligned}
& -\mathrm{P}_{0}\left(-i \Sigma_{\mathrm{f}_{-} \mathrm{se}}^{\mathrm{UV}}(0)\right)\left(\mathrm{IP}_{-1} \mathrm{O}+\mathrm{OP}_{1} \mathrm{I}\right)-\left(\mathrm{IP}_{-1} \mathrm{O}+\mathrm{OP}_{1} \mathrm{I}\right)\left(-i \Sigma_{\mathrm{f}_{-} \mathrm{se}}^{\mathrm{UV}}(0)\right) \mathrm{P}_{0} \text {. }
\end{aligned}
$$

Combining this vertex contribution with the simpler self-energy corrections gives the ultraviolet pole identification that

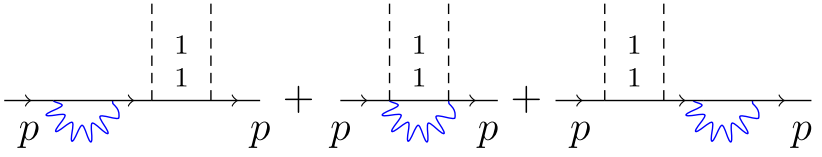

$$
\begin{aligned}
& \underset{\mathrm{UV}}{=} \mathrm{IP}_{-1}\left(-i \Sigma_{\mathrm{f}_{-\mathrm{se}}}^{\mathrm{UV}}(-1)\right) \mathrm{P}_{-1} \mathrm{O} \\
& +\mathrm{P}_{0}\left(-i \Sigma_{\mathrm{f}_{\text {se }}}^{\mathrm{UV}}(0)\right) \mathrm{P}_{0}(-i \boldsymbol{\mu}) \mathrm{P}_{0}+\mathrm{P}_{0}\left(-i \Sigma_{\mu}^{\mathrm{UV}}\right) \mathrm{P}_{0} \\
& +\mathrm{P}_{0}(-i \mu) \mathrm{P}_{0}\left(-i \Sigma_{\mathrm{f}_{-} \mathrm{se}}^{\mathrm{UV}}(0)\right) \mathrm{P}_{0} \\
& +\mathrm{OP}_{1}\left(-i \Sigma_{\mathrm{f}_{-s e}}^{\mathrm{UV}}(1)\right) \mathrm{P}_{1} \mathrm{I}
\end{aligned}
$$

As seen earlier for the simpler absorption and emission processes, this contains the expected propagator renormalization terms for each sideband. But, in addition, the middle term in the central sideband here shows the gauge dependent, ultraviolet divergence related to the background induced mass, where

$$
-i \Sigma_{\mathscr{M V}}^{\mathrm{UV}}=-i \frac{e^{2}}{(4 \pi)^{2}} \xi M \frac{1}{\varepsilon}
$$

The results presented in (81), (84), and (86) are the lowest order, ultraviolet corrections to the fundamental interactions of the electron with a circularly polarized but weak background. They show a sideband structure, which leads to a multiplicative renormalization at this order, as discussed in $[6,20]$. The use of the momentum gauge has greatly simplified this analysis. In the next section we consider the extension of these results to the strong field situation where results at all orders in the background field are needed.

\section{ONE-LOOP CORRECTION IN A STRONG BACKGROUND}

Extending the weak field, one-loop results to strong field QED will follow the basic steps seen earlier for scalar matter, but now this needs to be done within each of the sidebands. Thus the fermionic version of the scalar double line propagator, (33), will now involve an additional sum over all possible sidebands. In this section we will present the key steps in arriving at such a description of the fermion propagating through such an intense background. Some of the important technical details of this account will be collected together in Appendix D.

The ultraviolet factorization, seen in (29), was the key technical tool in our analysis of the one-loop results for scalar matter. We have already seen the tree-level version 
of this for fermionic matter in (64) and, in expression (D1) of Appendix D, we describe its one-loop version. Using it, we quickly see that the strong background interaction version of the absorption loop corrections, (81), can be written as

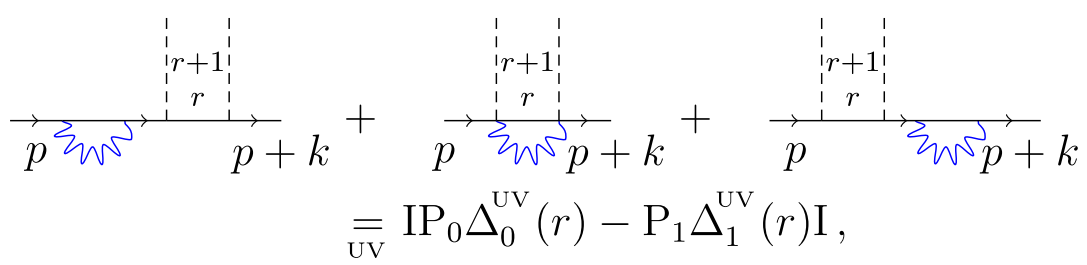

for $r \geq 0$. In this expression we have extended to fermions, and their sidebands, the notation, $\Delta^{\mathrm{UV}}(r)$, introduced for scalar matter in (31). Now the $n$th sideband at order $r$ has a factor $\Delta_{n}^{\mathrm{UV}}(r)$ in (88) where, if $r \geq 1$,

$$
\Delta_{n}^{\mathrm{UV}}(r):=\sum_{s=0}^{r}\left(-i \not l \mathrm{P}_{n}\right)^{s}\left(-i \Sigma_{\mathrm{f}_{-} \mathrm{se}}^{\mathrm{UV}}(n) \mathrm{P}_{n}\right)\left(-i \not l \mathrm{P}_{n}\right)^{r-s}+\sum_{s=0}^{r-1}\left(-i \not l \mathrm{P}_{n}\right)^{s}\left(-i \Sigma_{\not d}^{\mathrm{UV}} \mathrm{P}_{n}\right)\left(-i \not l \mathrm{P}_{n}\right)^{r-1-s}
$$

while if $r=0$,

$$
\Delta_{n}^{\mathrm{UV}}(0)=-i \Sigma_{\mathrm{f}_{\text {se }}}^{\mathrm{UV}}(n) \mathrm{P}_{n} .
$$

In a similar way, the higher order versions of the background corrections to the fundamental emission one-loop result, (84), is for all $r \geq 0$,

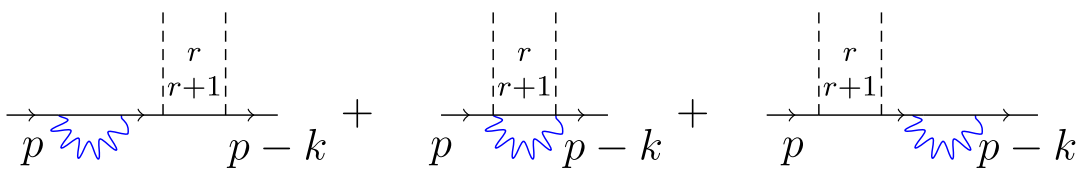

$$
\begin{aligned}
& \underset{\mathrm{UV}}{=} \mathrm{P}_{-1} \Delta_{-1}^{\mathrm{UV}}(r) \mathrm{O}-\mathrm{OP}_{0} \Delta_{0}^{\mathrm{UV}}(r) \text {. }
\end{aligned}
$$

For the central terms, (86), we get the generalization, but now for $r \geq 1$, that

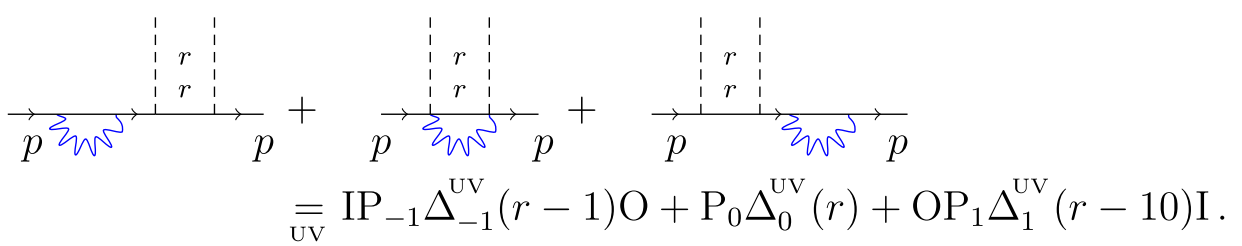

To this last result we also need to include the $r=0$, single self-energy correction, given by $\mathrm{P}_{0} \Delta_{0}^{\mathrm{UV}}(0)$.

We now sum over all such sideband terms. In such a sum we need to remember that expression (89) is only valid when $r \geq 1$. The first sum here, though, does make sense when $r=0$, and gives the correct result. The second sum in (89) only makes sense if $r \geq 1$, but can be easily shifted to also start at $r=0$. Thus we get

$$
\begin{aligned}
& \sum_{r=0}^{\infty} \mathrm{P}_{n} \Delta_{n}^{\mathrm{UV}}(r)=\sum_{r=0}^{\infty} \sum_{s=0}^{r} \mathrm{P}_{n}\left(-i \not l \mathrm{P}_{n}\right)^{s}\left(-i\left(\Sigma_{\mathrm{f}_{-} \mathrm{se}}^{\mathrm{UV}}(n)+\Sigma_{\mathscr{M}}^{\mathrm{UV}}\right)\right) \mathrm{P}_{n}\left(-i M \mathrm{P}_{n}\right)^{r-s} \\
& =\sum_{i=0}^{\infty} \mathrm{P}_{n}\left(-i \not l \mathrm{P}_{n}\right)^{i}\left(-i\left(\Sigma_{\mathrm{f}_{-} \mathrm{se}}^{\mathrm{UV}}(n)+\Sigma_{\mathscr{M}}^{\mathrm{UV}}\right)\right) \sum_{j=0}^{\infty} \mathrm{P}_{n}\left(-i \not \mathrm{P}_{n}\right)^{j} \\
& =\mathrm{P}(m+\mathscr{M})_{n}\left(-i\left(\Sigma_{\mathrm{f}_{-} \mathrm{se}}^{\mathrm{UV}}(n)+\Sigma_{\mathscr{M}}^{\mathrm{UV}}\right)\right) \mathrm{P}(m+\mathscr{M})_{n},
\end{aligned}
$$

where in the last line we have used the matrix mass shifted, propagator result (69). 
Applying this last result to the sums over the sideband expressions (88), (91), and (92), gives us the fermionic, double line, one-loop result that

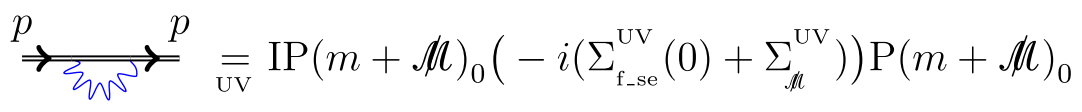

$$
\begin{aligned}
& -\mathrm{P}(m+\mu)_{1}\left(-i\left(\Sigma_{\mathrm{f}_{-} \mathrm{se}}^{\mathrm{UV}}(1)+\Sigma_{\mu}^{\mathrm{UV}}\right)\right) \mathrm{P}\left(m+\mu_{M}\right)_{1} \mathrm{I} \\
& +\mathrm{IP}(m+\mu)_{-1}\left(-i\left(\Sigma_{\mathrm{f}_{-} \mathrm{se}}^{\mathrm{UV}}(-1)+\Sigma_{\mu}^{\mathrm{UV}}\right)\right) \mathrm{P}(m+\boldsymbol{M})_{-1} \mathrm{O} \\
& +\mathrm{P}(m+\mu)_{0}\left(-i\left(\Sigma_{\mathrm{f}_{\mathrm{se}} \mathrm{UV}}^{\mathrm{UV}}(0)+\Sigma_{\mu}^{\mathrm{UV}}\right)\right) \mathrm{P}\left(m+\mu_{\mu}\right)_{0} \\
& +\mathrm{OP}(m+\mu)_{1}\left(-i\left(\Sigma_{\mathrm{f}_{-} \mathrm{se}}^{\mathrm{UV}}(1)+\Sigma_{\boldsymbol{M}}^{\mathrm{UV}}\right)\right) \mathrm{P}(m+\boldsymbol{M})_{1} \mathrm{I} \\
& +\mathrm{P}(m+\mathscr{M})_{-1}\left(-i\left(\Sigma_{\mathrm{f}_{-} \mathrm{se}}^{\mathrm{UV}}(-1)+\Sigma_{\boldsymbol{M}}^{\mathrm{UV}}\right)\right) \mathrm{P}\left(m+\boldsymbol{M}_{-1} \mathrm{O}\right. \\
& -\mathrm{OP}\left(m+\boldsymbol{M}_{0}\left(-i\left(\Sigma_{\mathrm{f}-\mathrm{se}}^{\mathrm{UV}}(0)+\Sigma_{\mathcal{M}}^{\mathrm{UV}}\right)\right) \mathrm{P}\left(m+\boldsymbol{M}_{0}\right)_{0} .\right.
\end{aligned}
$$

This last result, along with the tree-level definition of the fermionic double line propagator, (70), gives a precise and surprisingly succinct description of the propagation in our background, to which standard renormalization techniques can be applied.

\section{RENORMALIZATION IN A STRONG BACKGROUND}

Having identified the one-loop ultraviolet poles for both scalar and fermionic matter in our background, we can now introduce counterterms and discuss the renormalization of these theories. Surprisingly, we shall see that there are very simple interpretations of the renormalization process in both cases.

The one-loop corrections to the double line propagators, introduced here in Secs. III and VII, now need to be interpreted in terms of bare fields and then renormalised via the introduction of appropriate counterterms. In $[6,20]$, it was seen that, even in a weak background, an additional counterterm was needed to renormalize the fermionic theory. Having extended here these one-loop calculations, for both types of matter, to all orders in the background, we can now look more precisely at the set of counterterms needed to renormalize the two theories.

Starting with scalar matter, the expressions for the selfenergy, (25), and background induced mass correction, (28), need to be added together and properly interpreted before we introduce any counterterms. We can write these one-loop corrections as

$$
\begin{aligned}
- & i\left(\Sigma_{\mathrm{S}-\mathrm{se}}^{\mathrm{UV}}+\Sigma_{\mathfrak{m}^{2}}^{\mathrm{UV}}\right) \\
& =-i \frac{e^{2}}{(4 \pi)^{2}} \frac{1}{\varepsilon}\left(3 m^{2}-i(\xi-3) \mathrm{P}\left(m^{2}\right)^{-1}+\xi \mathfrak{m}^{2}\right) .
\end{aligned}
$$

The first thing to note about this expression is that the inverse propagator term here does not match the double line propagators that surround these corrections in (33). Under the simple mass shift, $\mathrm{P}\left(m^{2}\right)^{-1}=\mathrm{P}\left(m^{2}+\mathfrak{m}^{2}\right)^{-1}-i \mathfrak{m}^{2}$, we get the full inverse propagator plus additional $\mathrm{m}^{2}$ terms that now combine, in a very attractive way, with the mass term to give

$$
\begin{aligned}
- & i\left(\Sigma_{\mathrm{S}_{-} \mathrm{Ue}}^{\mathrm{UV}}+\Sigma_{\mathfrak{m}^{2}}^{\mathrm{UV}}\right) \\
& =-i \frac{e^{2}}{(4 \pi)^{2}} \frac{1}{\varepsilon}\left(3\left(m^{2}+\mathfrak{m}^{2}\right)-i(\xi-3) \mathrm{P}\left(m^{2}+\mathfrak{m}^{2}\right)^{-1}\right) .
\end{aligned}
$$

Note that this last expression can be interpreted as the usual ultraviolet pole of the self-energy for a scalar particle of mass $m_{*}^{2}=m^{2}+\mathfrak{m}^{2}$. Now introducing the tree-level, but not free, bare mass $m_{*}^{2}$ and the, Volkov field, wave function renormalization, allows for the expansion in terms of mass, $\delta_{m_{*}^{2}}$, and Volkov wave function, $\delta_{2}$, counterterms so that

$-i\left(\Sigma_{\mathrm{S}_{\_} \mathrm{Se}}^{\mathrm{UV}}+\Sigma_{\mathfrak{m}^{2}}^{\mathrm{UV}}\right) \rightarrow-i\left(\Sigma_{\mathrm{S}_{-} \mathrm{Se}}^{\mathrm{UV}}+\Sigma_{\mathfrak{m}^{2}}^{\mathrm{UV}}+m_{*}^{2} \delta_{m_{*}^{2}}-i \mathrm{P}\left(m_{*}^{2}\right)^{-1} \delta_{2}\right)$.

From (96) we can now read off the strong field renormalization conditions that

$$
\delta_{m_{*}^{2}}=-\frac{e^{2}}{(4 \pi)^{2}} \frac{3}{\varepsilon} \quad \text { and } \quad \delta_{2}=-(\xi-3) \frac{e^{2}}{(4 \pi)^{2}} \frac{1}{\varepsilon} .
$$

The most striking thing about this result is its simplicity. In terms of the strong field variables, $m_{*}^{2}$ and $\mathrm{P}\left(m_{*}^{2}\right)^{-1}$, we have the same multiplicative structure familiar from the scalar matter in a vacuum. If we were not in the momentum gauge, then many additional gauge artifacts would obstruct this simple result. 
For the fermionic theory, this argument needs to be repeated in each of the sidebands, with propagator $\mathrm{P}_{n}$, for $n$ either 0 or \pm 1 . We quickly see that for the $n$th such sideband,

$-i\left(\Sigma_{\mathrm{f}_{\text {se }}}^{\mathrm{UV}}(n)+\Sigma_{\mathscr{M}}^{\mathrm{UV}}\right)=-i \frac{e^{2}}{(4 \pi)^{2}} \frac{1}{\varepsilon}\left(3 m-i \xi \mathrm{P}(m)_{n}^{-1}+\xi \mathscr{M}\right)$.

Again, the inverse propagator here needs to match the terms multiplying it, so we make the replacement $\mathrm{P}(m)_{n}^{-1}=$ $\mathrm{P}(m+\mathscr{M})^{-1}-i \mathscr{M}$. The end result of this shift is that

$-i\left(\Sigma_{\mathrm{f}_{-} \mathrm{se}}^{\mathrm{UV}}(n)+\Sigma_{\mathscr{M}}^{\mathrm{UV}}\right)=-i \frac{e^{2}}{(4 \pi)^{2}} \frac{1}{\varepsilon}\left(3 m-i \xi \mathrm{P}(m+\mathscr{M})_{n}^{-1}\right)$.

In contrast to the scalar result above, now we see only a renormalization of the vacuum mass, $m$, but the wave function term is still with respect to the full, strong field normalization.

Thus, in this fermionic theory, we need to introduce just a vacuum mass counterterm, $\delta_{m}$, along with the strong field, wave function term, $\delta_{2}$. Then, written in terms of renormalized fields, we have

$$
\begin{aligned}
- & i\left(\Sigma_{\mathrm{f}_{\text {se }}}^{\mathrm{UV}}(n)+\Sigma_{\mathscr{M}}^{\mathrm{UV}}\right) \\
& \rightarrow-i\left(\Sigma_{\mathrm{f} \_\mathrm{se}}^{\mathrm{UV}}(n)+\Sigma_{\mathscr{M}}^{\mathrm{UV}}+m \delta_{m}-i \mathrm{P}(m+\mathscr{M})_{n}^{-1} \delta_{2}\right) .
\end{aligned}
$$

The renormalization conditions that follow from this and (96) are now

$$
\delta_{m}=-\frac{e^{2}}{(4 \pi)^{2}} \frac{3}{\varepsilon} \quad \text { and } \quad \delta_{2}=-\xi \frac{e^{2}}{(4 \pi)^{2}} \frac{1}{\varepsilon} .
$$

The differences revealed in this section in the renormalization conditions needed for scalar, (98), and fermionic, (102), matter are surprising. That the gauge fixing conditions enter differently is not itself unexpected, but the fact that different classes of counterterms are needed seems unexpected. In particular, the contrast between the full mass counterterm for scalars and only the vacuum mass counterterm for fermions seems unexpected. It is not clear, a priori, why this should be the case. Especially given the fact that both types of matter have the same, strong field, counterterm structure for the wave function renormalization associated with the full propagators (19) and (70).

\section{CONCLUSIONS}

There is huge theoretical and experimental interest in particle physics in an intense laser background. Much of the theoretical work builds upon the Volkov solution, where there is known to be a mass shift and a loss of translational invariance, both induced by the background. The Volkov solution has built into it a gauge freedom. In this paper we have introduced an additional gauge fixing condition on the background, which we call the momentum gauge, which dramatically simplifies the description of charged matter propagation.

For scalar matter, in a circularly polarized background, we have seen in this paper that only one type of background interaction survives in this gauge. This interaction respects translation invariance, and can be easily summed to all orders. As the background nontranslational invariance has been gauged away, all the familiar tools from vacuum scalar QED could be deployed. The strong field solution developed here exhibits the background induced mass shift, but none of the normally expected sideband structures, which are revealed here to be gauge artifacts, even at one-loop.

For fermionic matter, a small number of sidebands persist in the momentum gauge. This corresponds to a limited violation of translational invariance. Despite this, we have been able to develop momentum space techniques to construct the propagator and its one-loop, ultraviolet corrections. The background induced mass term has a matrix structure that is common to each sideband. We emphasize that in this momentum gauge, the infinite tower of sidebands has reduced to just seven terms for fermionic matter, to be compared to just one for the scalar theory. This reinforces our observation that the usual infinite number of sidebands is a gauge artifact of this theory.

Our analysis of the renormalization has further revealed a difference in the counterterm structures needed in both theories. For the scalar matter, (96), we have renormalization of the shifted mass for the full propagator, and of the residue of the pole at this shifted mass. For fermionic matter, (100), the vacuum mass is renormalized, rather than the shifted mass, but we saw that it is the residue of the shifted mass that acquires a wave function renormalization.

In this paper we have considered tree-level and the ultraviolet one-loop contributions to charge propagation. Future work will include an analysis of the finite parts and the on-shell infrared structures associated with charged matter propagating in the plane wave background. The intriguing issue to be resolved for the infrared is the significance of going on-shell at either the shifted or vacuum mass. Also, the simple form of our propagator may shed further light on the asymptotic behavior of QED, [21-23].

In describing charge propagation, there was a natural momentum that could be used to simplify our description of propagation, as presented above. In a scattering situation, see for example [24], one can only expect to simplify one leg of the process by matching the gauge choice to the momentum in that leg. The implications of this simplification for scattering, and how gauge transformations can shift which leg has been trivialized, will be explored elsewhere. 
Our focus on circular polarization for the background field has led to particularly simple results for both the tree-level and one-loop renormalization of these theories. The most immediate impact of widening the class of polarizations is that the terms $v$ and $v^{*}$, as defined in (44), no longer vanish, and one will get Bessel functions of these terms as factors in the sideband structure. This was alluded to in Eq. (B2) of this paper. These added effects from the background will impact on our results for both scalar and fermionic matter. However, the evidence from $[6,20]$ is that these terms do not acquire any one-loop corrections. We conjecture that this observation will also hold in a strong background for both types of matter. The circular polarization case considered in this paper seems to represent the simplest configuration that captures the essential physics of the loop corrections in a plane wave background, for both types of matter.

\section{ACKNOWLEDGMENTS}

We thank Tom Heinzl for discussions.

\section{APPENDIX A: PERTURBATIVE FACTORIZATION RESULTS}

In this appendix we collect together the details of the arguments that lead to the key perturbative factorization result (64), and then the explicit expressions (65), (66), and (67) that follow from it. The simplicity of these results all depend critically on our choice of gauge and polarization.

Although the factorization result has been stated quite generally in (64), the vanishing result (61) means that we only need to consider three nontrivial cases corresponding to a net absorption $\left(r_{2}=r_{1}-1\right)$, a balanced interaction $\left(r_{2}=r_{1}\right)$, or a net emission $\left(r_{2}=r_{1}+1\right)$.

A net absorption means that there will be one extra power of the absorption vertex (34) over the emission vertex (35). The vanishing results (47) means that these vertices must alternate and hence, for $r \geq 0$,

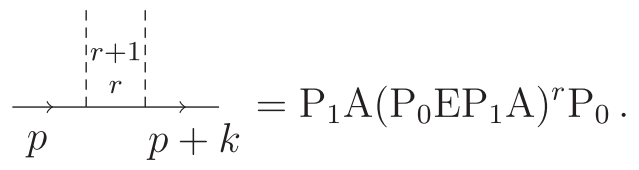

If $r=0$ this reduces to the fundamental absorption process (58), while if $r>0$ we have, using the vanishing identities (47),

$$
\begin{aligned}
& \mathrm{P}_{1} \mathrm{~A}\left(\mathrm{P}_{0} \mathrm{EP}_{1} \mathrm{~A}\right)^{r} \mathrm{P}_{0} \\
& =\mathrm{P}_{1} \mathrm{~A}\left(\mathrm{P}_{0} \mathrm{EP}_{1} \mathrm{~A}\right)^{r-1}\left(\mathrm{P}_{0} \mathrm{EP}_{1} \mathrm{~A}+\mathrm{P}_{0} \mathrm{AP}_{-1} \mathrm{E}\right) \mathrm{P}_{0} \\
& =\mathrm{P}_{1} \mathrm{~A}\left(\mathrm{P}_{0} \mathrm{EP}_{1} \mathrm{~A}\right)^{r-1} \mathrm{P}_{0}\left(\mathrm{EP}_{1} \mathrm{~A}+\mathrm{AP}_{-1} \mathrm{E}\right) \mathrm{P}_{0}
\end{aligned}
$$

Hence we recover the factorization identity that, for $r>0$,

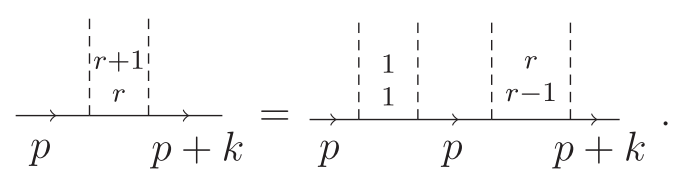

The dual emission version of this factorization identity can be shown in a very similar way. For the balanced case there are now two contributing terms when we have $r>0$ absorptions and emissions: $\left(\mathrm{P}_{0} \mathrm{EP}_{1} \mathrm{~A}\right)^{r} \mathrm{P}_{0}+\left(\mathrm{P}_{0} \mathrm{AP}_{-1} \mathrm{E}\right)^{r} \mathrm{P}_{0}$. But, using the vanishing identities again, this can be written as $\left(\mathrm{P}_{0} \mathrm{EP}_{1} \mathrm{~A}+\mathrm{P}_{0} \mathrm{AP}_{-1} \mathrm{E}\right)^{r} \mathrm{P}_{0}$, from which the factorization result immediately follows.

These factorization results now allow for an inductive derivation of the key identities (65), (66), and (67), where the base cases have already been seen in (58), (59), and (63). In fact, we only need to show (67), as the other two then follow using repeated applications of the factorization results.

Assuming the identity (67) holds for $r \geq 1$ absorptions and emissions, the factorization result then allows us to write

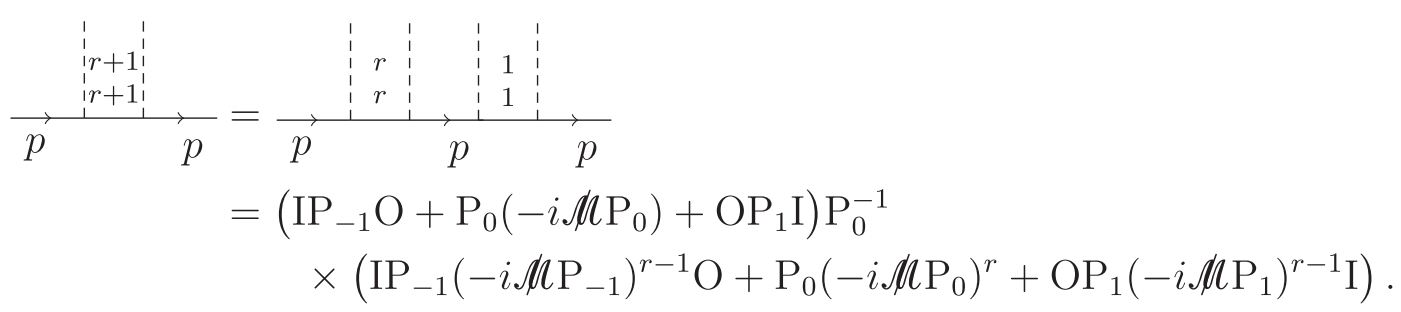

Expanding the right-hand side of (A4) yields nine potential terms but this quickly reduces to three by using the trivial identities that $\mathscr{M} \mathrm{I}=\mathscr{M O}=0$, along with (51). Thus we get

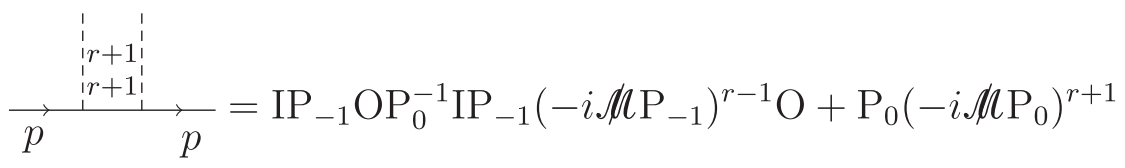

$$
\begin{aligned}
& +\mathrm{OP}_{1} \mathrm{IP}_{0}^{1} \mathrm{OP}_{1}\left(-i \mu \mathrm{P}_{1}\right)^{r-1} \mathrm{I} \text {. }
\end{aligned}
$$


Now we can use the identities (55) and (56) to deduce the claimed result (67), for all $r \geq 0$.

\section{APPENDIX B: DERIVATION OF RITUS MATRICES}

The compact tree-level result (70) is of interest in its own right since there are other approaches to this double line propagator that are not based on perturbation theory, and look very different. As a check of the results developed here, we now show how the more familiar Ritus matrices [25] reduce to the terms in (70) for our choice of circular polarization and use of the momentum gauge.

In order to trace the consequences of the assumptions made in this paper, we first consider the more general elliptic class of polarizations, which includes circular and linear polarization as limiting cases. In Eq. (44) of [7], a suitable time-ordered product of Volkov fields was calculated and, written here in terms of a double line, shown to be equal to

$$
\stackrel{p}{\longrightarrow}=\sum_{n, r} \mathrm{e}^{i(n+r) x \cdot k} J_{n+r}\left(\Omega_{1}^{\prime}, v^{\prime}, \Omega_{2}^{\prime}\right) \mathrm{P}(m+\mu)_{n} \bar{J}_{n}\left(\Omega_{1}^{\prime}, v^{\prime}, \Omega_{2}^{\prime}\right) \mathrm{e}^{-i n x \cdot k} .
$$

The notation used here is more refined than that used in [7], and is essentially found in the discussion leading to Eq. (66) of [6]. The normalizing functions, $J_{n}$, are the elliptic class of generalized Bessel functions with first and last arguments $\Omega_{1}^{\prime}=-\left(\mathrm{I}^{\prime}+\mathrm{O}^{\prime}\right)$ and $\Omega_{2}^{\prime}=-i\left(\mathrm{I}^{\prime}-\mathrm{O}^{\prime}\right)$. The "In" and "Out" terms here are written with primes to signify that they do not include the final exponential factors in the complex potential (2), used in this paper. But we note that the terms in (B1) are multiplied by the exponential $\mathrm{e}^{i r x \cdot k}$, which we have factorized to match the order of the normalizing Bessel functions. These factors can then be reabsorbed into the arguments of the Bessel functions, with the result that the parameters shift from functions of $\mathrm{I}^{\prime}$ and $\mathrm{O}^{\prime}$ to those of I and $\mathrm{O}$, as used in this paper. In the same way, the scalar $v^{\prime}$ becomes the $v$ of Eq. (44), which only vanishes for circular polarization. By shifting the background induced mass term out of the propagator in (B1), one recovers the Ritus representation of the double line propagator. See the discussion around Eq. (35) in [7] for more details.

We now start to restrict this quite general representation for the double line propagator to the situation considered in this paper. The first thing to note is that in the momentum gauge the generalized Bessel functions can be easily expanded in terms of the "In" and "Out" matrices resulting in the simplification that

$$
\begin{aligned}
J_{n}( & -(\mathrm{I}+\mathrm{O}), v,-i(\mathrm{I}-\mathrm{O})) \\
= & \begin{cases}J_{(n+1) / 2}(v) \mathrm{I}-J_{(n-1) / 2}(v) \mathrm{O}, & \text { if } n \text { is odd } \\
J_{n / 2}(v), & \text { if } n \text { is even, }\end{cases}
\end{aligned}
$$

where the right-hand side now involves just standard Bessel functions.

If we now impose the circular polarization condition that $v=0$, then these Bessel functions further simplify so that only three terms survive:

$$
\begin{aligned}
& J_{-1}(-(\mathrm{I}+\mathrm{O}), 0,-i(\mathrm{I}-\mathrm{O}))=\mathrm{I}, \\
& J_{0}(-(\mathrm{I}+\mathrm{O}), 0,-i(\mathrm{I}-\mathrm{O}))=1,
\end{aligned}
$$

and

$$
J_{1}(-(\mathrm{I}+\mathrm{O}), 0,-i(\mathrm{I}-\mathrm{O}))=-\mathrm{O}
$$

Inserting these results into the expression (B1) then quickly recovers the result (70) for the double line propagator that was derived perturbatively in this paper.

\section{APPENDIX C: INDUCED MASS LOOP CORRECTION}

In this appendix we wish to sketch the key steps in deriving the one-loop result (85) and hence the background induced mass loop correction (87).

We have seen that the background induced mass arises from the mixture of absorptions and emissions to the background. The expression underlying this sideband result (63) is

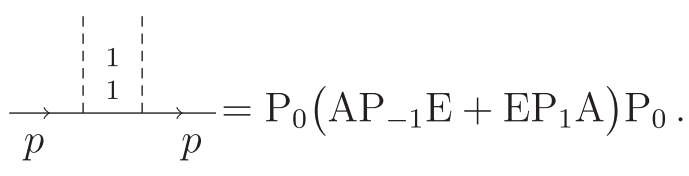

Following the discussion related to Fig. 9 in [6], the ultraviolet pole of the one-loop version of the bracketed expression on the right in $(\mathrm{C} 1)$ can easily be found by the simple algebraic replacement: $\mathrm{P}_{n} \rightarrow \mathrm{P}_{n}+\mathrm{P}_{n}\left(-i \Sigma_{\text {f.se }}^{\mathrm{UV}}(n)\right) \mathrm{P}_{n}$; $\mathrm{A} \rightarrow \mathrm{A}-i \Sigma_{\text {in }}^{\mathrm{UV}}$ and $\mathrm{E} \rightarrow \mathrm{E}-i \Sigma_{\text {out }}^{\mathrm{UV}}$. Hence we have the one-loop ultraviolet pole identification that 


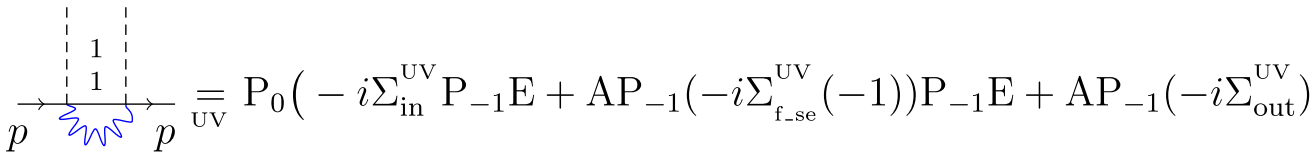

$$
\begin{aligned}
& -i \Sigma_{\text {out }}^{\mathrm{UV}} \mathrm{P}_{1} \mathrm{~A}+\mathrm{EP}_{1}\left(-i \Sigma_{\mathrm{f}_{-} \mathrm{se}}^{\mathrm{UV}}(1) \mathrm{P}_{1} \mathrm{~A}+\mathrm{EP}_{1}\left(-i \Sigma_{\text {in }}^{\mathrm{UV}}\right)\right) \mathrm{P}_{0} .
\end{aligned}
$$

These can now be evaluated in terms of sideband loop corrections using the tree-level identities in (41) and the vertex results (80) and (83).

One quickly finds that

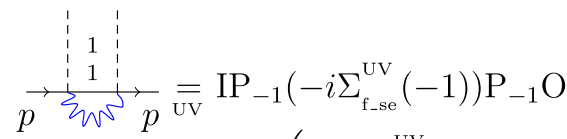

$$
\begin{aligned}
& -\mathrm{P}_{0}\left(\mathrm{I}\left(-i \Sigma_{\mathrm{f}_{\mathrm{se}}}^{\mathrm{UV}}(-1)\right) \mathrm{O}+\mathrm{O}\left(-i \Sigma_{\mathrm{f}_{-s e}}^{\mathrm{UV}}(1)\right) \mathrm{I}\right) \mathrm{P}_{0} \\
& +\mathrm{OP}_{1}\left(-i \Sigma_{\mathrm{f}_{\mathrm{ses}}}^{\mathrm{UV}}(1)\right) \mathrm{P}_{1} \mathrm{I} \\
& -\mathrm{P}_{0}\left(-i \Sigma_{\mathrm{f}_{-} \mathrm{se}}^{\mathrm{UV}}(0)\right)\left(\mathrm{IP}_{-1} \mathrm{O}+\mathrm{OP}_{1} \mathrm{I}\right) \\
& -\left(\mathrm{IP}_{-1} \mathrm{O}+\mathrm{OP}_{1} \mathrm{I}\right)\left(-i \Sigma_{\mathrm{f}-\mathrm{se}}^{\mathrm{UV}}(0)\right) \mathrm{P}_{0} \text {. }
\end{aligned}
$$

Now using the results (55) and (75), we see that

$\mathrm{I}\left(-i \Sigma_{\mathrm{f}_{-} \mathrm{se}}^{\mathrm{UV}}(-1)\right) \mathrm{O}+\mathrm{O}\left(-i \Sigma_{\mathrm{f}_{-} \mathrm{se}}^{\mathrm{UV}}(1)\right) \mathrm{I}=\frac{i e^{2}}{(4 \pi)^{2}} \xi M \frac{1}{\varepsilon}$.

Combining this last expression with the expansion (C3) then gives the key result (85) in the main text.

\section{APPENDIX D: SOME STRONG FIELD RESULTS}

The tree-level, fermionic factorization identity (64) immediately gives the ultraviolet, one-loop, factorization result that

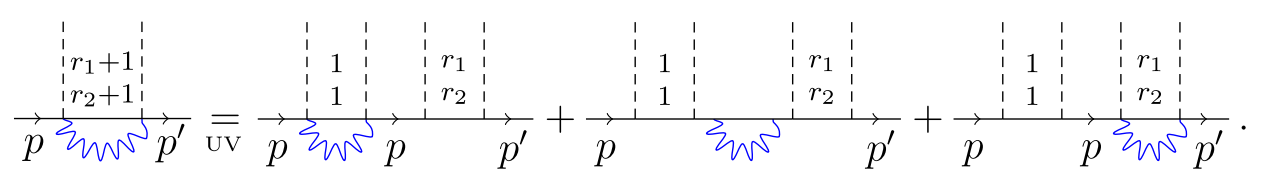

This now allows us to inductively build up the one-loop corrections to all the higher order interactions with the background.

For the central terms, where $r_{1}=r_{2}$, we now use this ultraviolet factorization result to prove that, for all $r \geq 1$,

$$
\begin{aligned}
& p \sum_{n} \sum_{p}=\mathrm{IP}_{-1} \Delta_{-1}^{\mathrm{UV}}(r-1) \mathrm{O}+\mathrm{P}_{0} \Delta_{0}^{\mathrm{UV}}(r)+\mathrm{OP}_{1} \Delta_{1}^{\mathrm{UV}}(r-1) \mathrm{I} \\
& -\left(\mathrm{IP}_{-1}\left(-i \mu \mathrm{P}_{-1}\right)^{r-1} \mathrm{O}+\mathrm{P}_{0}\left(-i \mu \mathrm{P}_{0}\right)^{r}+\mathrm{OP}_{1}\left(-i \mu \mathrm{P}_{1}\right)^{r-1} \mathrm{I}\right)\left(-i \Sigma_{\mathrm{f}_{-} \mathrm{se}}^{\mathrm{UV}}(0)\right) \mathrm{P}_{0} \\
& -\mathrm{P}_{0}\left(-i \Sigma_{\mathrm{f}_{-} \mathrm{se}}^{\mathrm{UV}}(0)\right)\left(\mathrm{IP}_{-1}\left(-i \mu \mathrm{P}_{-1}\right)^{r} \mathrm{O}+\mathrm{P}_{0}\left(-i \mu \mathrm{P}_{0}\right)^{r+1}+\mathrm{OP}_{1}\left(-i \mu \mathrm{P}_{1}\right)^{r} \mathrm{I}\right) \text {, }
\end{aligned}
$$

which is equivalent to (92).

When $r=1$, this expression reduces to our earlier one-loop calculation, (85). To then show the result when we have $r+1$ absorptions and emissions, we use the factorization identity to disentangle the interactions, thus reducing to three processes where we have less interactions spanned by the loop, and hence the inductive assumption can be used.

So the key factorization result we need is that

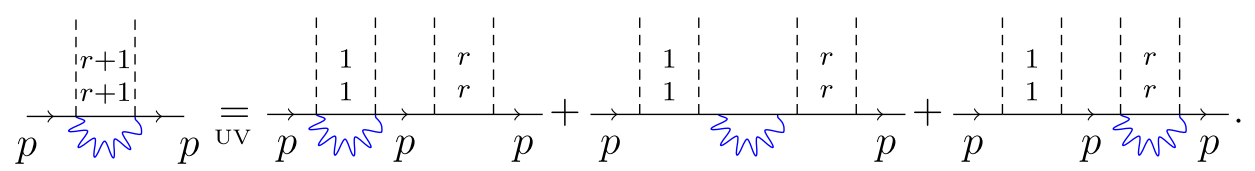

The second two diagrams here can be combined and simplified, after repeated use of the identities (56), to give 


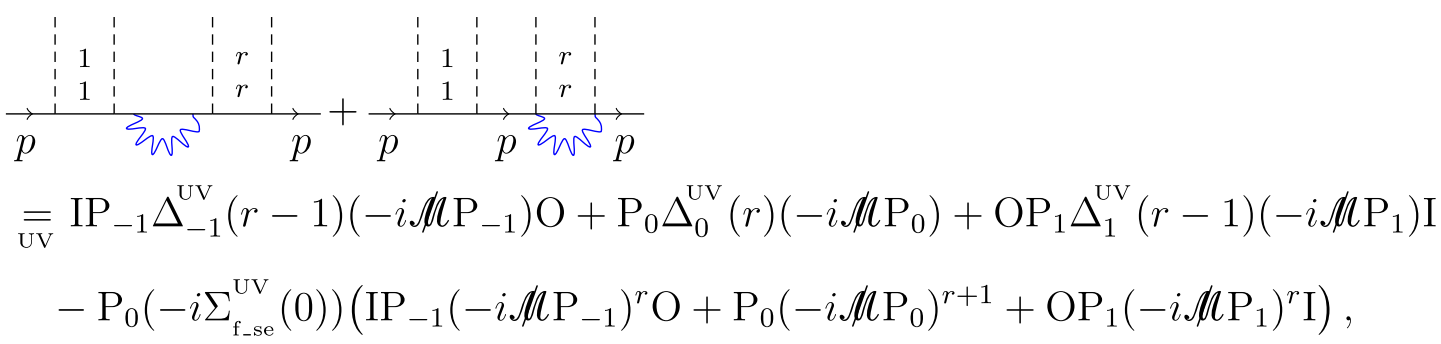

From the definition (89), we quickly see that

$$
\Delta_{n}^{\mathrm{UV}}(r)\left(-i \not l \mathrm{P}_{n}\right)=\Delta_{n}^{\mathrm{UV}}(r+1)-\left(-i \not l \mathrm{P}_{n}\right)^{r+1}\left(-i \Sigma_{\mathrm{f}_{-} \mathrm{se}}^{\mathrm{UV}}(n) \mathrm{P}_{n}\right)-\left(-i \not l \mathrm{P}_{n}\right)^{r}\left(-i \Sigma_{\mathscr{M}}^{\mathrm{UV}} \mathrm{P}_{n}\right) .
$$

Using this result in (D4), we find that

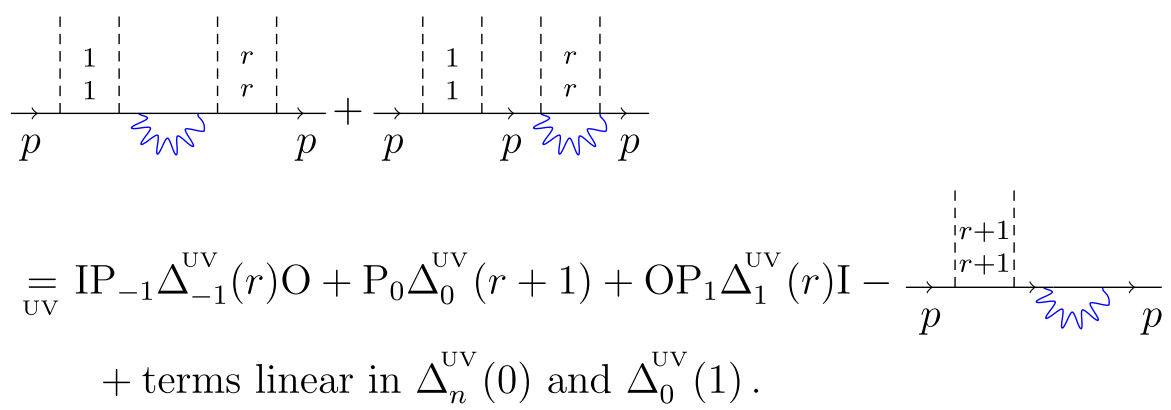

When the loop straddles the initial absorption and emission process, we get

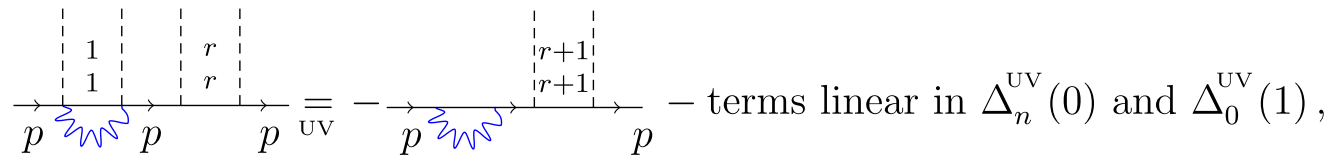

where the final terms here are, up to a sign, the same as in (D6).

Combining the expressions (D6) and (D7), we see that

$$
\begin{aligned}
& p \sum_{m} \sum_{p}=\mathrm{IP}_{-1} \Delta_{-1}^{\mathrm{UV}}(r) \mathrm{O}+\mathrm{P}_{0} \Delta_{0}^{\mathrm{UV}}(r+1)+\mathrm{OP}_{1} \Delta_{1}^{\mathrm{UV}}(r) \mathrm{I}
\end{aligned}
$$

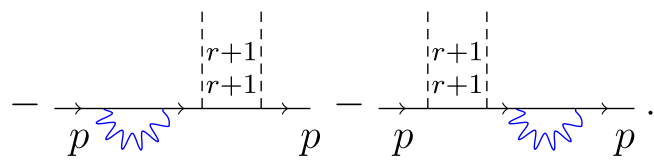

Thus the one-loop, central sideband result (D2) holds for all $r \geq 1$.

Armed with this central result, we can rapidly derive the expressions for the upper and lower sidebands, (88) and (91), by using, for example, the ultraviolet, one-loop factorization

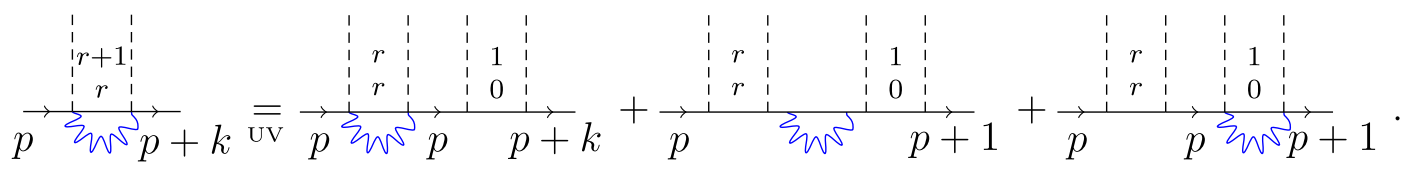


[1] P. A. M. Dirac, The quantum theory of the emission and absorption of radiation, Proc. R. Soc. Ser. A 114, 243 (1927).

[2] E. Fermi, Quantum theory of radiation, Rev. Mod. Phys. 4, 87 (1932).

[3] D. M. Volkov, Über eine Klasse von Lösungen der Diracschen Gleichung., Z. Phys. 94, 250 (1935).

[4] V. N. Baier, V. M. Katkov, A. I. Milshtein, and V. M. Strakhovenko, On the theory of quantum processes in the field of intense electromagnetic wave, Zh. Eksp. Teor. Fiz. 69, 783 (1975), http://jetp.ac.ru/cgi-bin/dn/e_042_03_0400 .pdf.

[5] V. N. Baier, V. M. Katkov, and V. M. Strakhovenko, An operator approach to quantum electrodynamics in external field: Mass operator, Zh. Eksp. Teor. Fiz. 67, 453 (1974), http://www.jetp.ac.ru/cgi-bin/dn/e_040_02_0225.pdf.

[6] M. Lavelle and D. McMullan, Renormalisation of the Volkov propagator, Phys. Rev. D 100, 036001 (2019).

[7] M. Lavelle and D. McMullan, Electrons in an eccentric background field, Phys. Rev. D 97, 036013 (2018).

[8] V. Dinu, T. Heinzl, A. Ilderton, M. Marklund, and G. Torgrimsson, Vacuum refractive indices and helicity flip in strong-field QED, Phys. Rev. D 89, 125003 (2014).

[9] S. Meuren, C. H. Keitel, and A. Di Piazza, Polarization operator for plane-wave background fields, Phys. Rev. D 88, 013007 (2013).

[10] B. King and T. Heinzl, Measuring vacuum polarisation with high power lasers High Power Laser Sci. Eng. 4, e5 (2016).

[11] G. F. Sterman, An Introduction to Quantum Field Theory (Cambridge University Press, Cambridge, England, 1993).

[12] C. Itzykson and J. B. Zuber, Quantum Field Theory, International Series In Pure and Applied Physics (McGraw-Hill, New York, 1980).

[13] V. B. Berestetskii, E. M. Lifshitz, and L. P. Pitaevskii, Quantum Electrodynamics, Volume 4 of Course of Theoretical Physics (Pergamon Press, Oxford, 1982).
[14] C. Bamber et al., Studies of nonlinear QED in collisions of 46.6-GeV electrons with intense laser pulses, Phys. Rev. D 60, 092004 (1999).

[15] T. Heinzl, D. Seipt, and B. Kampfer, Beam-shape effects in nonlinear Compton and Thomson scattering, Phys. Rev. A 81, 022125 (2010).

[16] N. D. Sengupta, On the scattering of electromagnetic waves by a free electron. II. Wave mechanical theory, Bull. Math. Soc. (Calcutta) 44, 175 (1952).

[17] M. Lavelle and D. McMullan, Fermionic propagator in an intense background., Phys. Rev. D 91, 105022 (2015).

[18] H. R. Reiss and J. H. Eberly, Green's function in intensefield electrodynamics, Phys. Rev. 151, 1058 (1966).

[19] J. H. Eberly and H. R. Reiss, Electron self-energy in intense plane-wave field, Phys. Rev. 145, 1035 (1966).

[20] M. Lavelle and D. McMullan, One loop Volkov propagator in the Lorentz class of gauges, Phys. Lett. B 798, 135021 (2019).

[21] T. Podszus and A. Di Piazza, High-energy behavior of strong-field QED in an intense plane wave, Phys. Rev. D 99, 076004 (2019).

[22] J. P. Edwards and A. Ilderton, Resummation of backgroundcollinear corrections in strong field QED, Phys. Rev. D 103, 016004 (2021).

[23] A. A. Mironov, S. Meuren, and A. M. Fedotov, Resummation of QED radiative corrections in a strong constant crossed field, Phys. Rev. D 102, 053005 (2020).

[24] A. Di Piazza and M. A. Lopez-Lopez, One-loop vertex correction in a plane wave, Phys. Rev. D 102, 076018 (2020).

[25] V. I. Ritus, Radiative corrections in quantum electrodynamics with intense field and their analytical properties, Ann. Phys. (N.Y.) 69, 555 (1972). 